\title{
Temperature and chemical species distributions in the middle atmosphere observed during Titan's late northern spring to early summer ${ }^{\star}$
}

\author{
S. Vinatier ${ }^{1}$, C. Mathé ${ }^{1,2}$, B. Bézard ${ }^{1}$, J. Vatant d'Ollone ${ }^{3,4}$, S. Lebonnois ${ }^{3}$, C. Dauphin ${ }^{5}$, F. M. Flasar ${ }^{6}$, \\ R. K. Achterberg ${ }^{6}$, B. Seignovert ${ }^{7}$, M. Sylvestre ${ }^{8}$, N. A. Teanby ${ }^{8}$, N. Gorius ${ }^{6}$, A. Mamoutkine ${ }^{9}$, \\ E. Guandique ${ }^{10}$, and D. E. Jennings ${ }^{6}$
}

(Affiliations can be found after the references)

Received 13 May 2020 / Accepted 19 June 2020

\begin{abstract}
We present a study of the seasonal evolution of Titan's thermal field and distributions of haze, $\mathrm{C}_{2} \mathrm{H}_{2}, \mathrm{C}_{2} \mathrm{H}_{4}, \mathrm{C}_{2} \mathrm{H}_{6}, \mathrm{CH}_{3} \mathrm{C}_{2} \mathrm{H}, \mathrm{C}_{3} \mathrm{H}_{8}$, $\mathrm{C}_{4} \mathrm{H}_{2}, \mathrm{C}_{6} \mathrm{H}_{6}, \mathrm{HCN}$, and $\mathrm{HC}_{3} \mathrm{~N}$ from March $2015\left(L_{s}=66^{\circ}\right)$ to September $2017\left(L_{s}=93^{\circ}\right)$ (i.e., from the last third of northern spring to early summer). We analyzed thermal emission of Titan's atmosphere acquired by the Cassini Composite Infrared Spectrometer with limb and nadir geometry to retrieve the stratospheric and mesospheric temperature and mixing ratios pole-to-pole meridional cross sections from 5 mbar to $50 \mu$ bar $(120-650 \mathrm{~km})$. The southern stratopause varied in a complex way and showed a global temperature increase from 2015 to 2017 at high-southern latitudes. Stratospheric southern polar temperatures, which were observed to be as low as $120 \mathrm{~K}$ in early 2015 due to the polar night, showed a $30 \mathrm{~K}$ increase (at $0.5 \mathrm{mbar}$ ) from March 2015 to May 2017 due to adiabatic heating in the subsiding branch of the global overturning circulation. All photochemical compounds were enriched at the south pole by this subsidence. Polar cross sections of these enhanced species, which are good tracers of the global dynamics, highlighted changes in the structure of the southern polar vortex. These high enhancements combined with the unusually low temperatures $(<120 \mathrm{~K})$ of the deep stratosphere resulted in condensation at the south pole between 0.1 and 0.03 mbar $(240-280 \mathrm{~km})$ of $\mathrm{HCN}_{2} \mathrm{HC}_{3} \mathrm{~N}, \mathrm{C}_{6} \mathrm{H}_{6}$ and possibly $\mathrm{C}_{4} \mathrm{H}_{2}$ in March $2015\left(L_{s}=66^{\circ}\right)$. These molecules were observed to condense deeper with increasing distance from the south pole. At high-northern latitudes, stratospheric enrichments remaining from the winter were observed below $300 \mathrm{~km}$ between 2015 and May $2017\left(L_{s}=90^{\circ}\right)$ for all chemical compounds and up to September $2017\left(L_{s}=93^{\circ}\right)$ for $\mathrm{C}_{2} \mathrm{H}_{2}, \mathrm{C}_{2} \mathrm{H}_{4}, \mathrm{CH}_{3} \mathrm{C}_{2} \mathrm{H}, \mathrm{C}_{3} \mathrm{H}_{8}$, and $\mathrm{C}_{4} \mathrm{H}_{2}$. In September 2017, these local enhancements were less pronounced than earlier for $\mathrm{C}_{2} \mathrm{H}_{2}, \mathrm{C}_{4} \mathrm{H}_{2}, \mathrm{CH}_{3} \mathrm{C}_{2} \mathrm{H}, \mathrm{HC} \mathrm{C}_{3} \mathrm{~N}$, and $\mathrm{HCN}$, and were no longer observed for $\mathrm{C}_{2} \mathrm{H}_{6}$ and $\mathrm{C}_{6} \mathrm{H}_{6}$, which suggests a change in the northern polar dynamics near the summer solstice. These enhancements observed during the entire spring may be due to confinement of this enriched air by a small remaining winter circulation cell that persisted in the low stratosphere up to the northern summer solstice, according to predictions of the Institut Pierre Simon Laplace Titan Global Climate Model (IPSL Titan GCM). In the mesosphere we derived a depleted layer in $\mathrm{C}_{2} \mathrm{H}_{2}, \mathrm{HCN}$, and $\mathrm{C}_{2} \mathrm{H}_{6}$ from the north pole to mid-southern latitudes, while $\mathrm{C}_{4} \mathrm{H}_{2}, \mathrm{C}_{3} \mathrm{H}_{4}, \mathrm{C}_{2} \mathrm{H}_{4}$, and $\mathrm{HC}_{3} \mathrm{~N}$ seem to have been enriched in the same region. In the deep stratosphere, all molecules except $\mathrm{C}_{2} \mathrm{H}_{4}$ were depleted due to their condensation sink located deeper than 5 mbar outside the southern polar vortex. $\mathrm{HCN}, \mathrm{C}_{4} \mathrm{H}_{2}$, and $\mathrm{CH}_{3} \mathrm{C}_{2} \mathrm{H}$ volume mixing ratio cross section contours showed steep slopes near the mid-latitudes or close to the equator, which can be explained by upwelling air in this region. Upwelling is also supported by the cross section of the $\mathrm{C}_{2} \mathrm{H}_{4}$ (the only molecule not condensing among those studied here) volume mixing ratio observed in the northern hemisphere. We derived the zonal wind velocity up to mesospheric levels from the retrieved thermal field. We show that zonal winds were faster and more confined around the south pole in $2015\left(L_{s}=67-72^{\circ}\right)$ than later. In 2016 , the polar zonal wind speed decreased while the fastest winds had migrated toward low-southern latitudes.
\end{abstract}

Key words. planets and satellites: individual: Titan - planets and satellites: atmospheres - planets and satellites: composition methods: data analysis - radiative transfer - infrared: planetary systems

\section{Introduction}

Because of the $26.7^{\circ}$ obliquity of Saturn, its system including Titan experiences strong seasonal changes. The Cassini mission arrived at the Saturn system on July 1, $2004\left(L_{s}=293^{\circ}\right)$, during the middle of Saturn's northern winter and ended on September $15,2017\left(L_{s}=93^{\circ}\right)$, a few months after the northern summer solstice. Titan Global Climate Models (GCMs) predict that during northern winter the global dynamics of Titan's middle atmosphere (from the deep stratosphere to the mesosphere) consists

\footnotetext{
* The data are only available at the CDS via anonymous ftp to cdsarc.u-strasbg.fr $(130.79 .128 .5)$ or via http://cdsarc. u-strasbg.fr/viz-bin/cat/J/A+A/641/A116
}

of one global circulation cell upwelling at high-southern latitudes and subsiding at high-northern latitudes (Newman et al. 2011; Lebonnois et al. 2012, 2014; Lora et al. 2015; Vatant d'Ollone et al. 2018). Impacts of this predicted dynamics were observed on the temperature field derived from the Cassini Composite Infrared Spectrometer (CIRS) observations of Titan's atmosphere thermal emission. The highest mesospheric temperatures, which were derived above the winter pole (Flasar et al. 2005; Achterberg et al. 2008, 2011; Coustenis et al. 2010; Mathé et al. 2020; Teanby et al. 2007, 2008; Vinatier et al. 2007, 2010a), resulted from adiabatic heating of the subsiding branch of the pole-to-pole circulation cell. Additionally, this polar subsidence transported the air enriched in photochemical compounds 
produced at higher altitude levels towards the deep stratosphere where some molecules such as $\mathrm{HC}_{3} \mathrm{~N}$ or $\mathrm{C}_{4} \mathrm{H}_{2}$ were observed to be enhanced by several orders of magnitude compared to their values outside the winter polar vortex (Coustenis et al. 2007; Mathé et al. 2020; Teanby et al. 2007, 2008; Vinatier et al. 2007, 2010a). Around the northern spring equinox, which occurred on August 11, $2009\left(L_{s}=0^{\circ}\right)$, GCM predict changes of this dynamical pattern with a two-year transition period involving a two-cell circulation regime with air upwelling at low latitudes and subsidence at both poles. Achterberg et al. (2011), who derived the seasonal evolution of the temperature field from July 2004 to December $2012\left(L_{s}\right.$ from $293^{\circ}$ to $\left.4^{\circ}\right)$ using CIRS spectra, showed that the subsiding branch at the north pole had already weakened before the northern spring equinox. In the early southern fall, the first southern polar molecular enhancements, derived from CIRS limb spectra analysis, were observed in June 2011 $\left(L_{s}=22.7^{\circ}\right)$ at altitudes higher than $400 \mathrm{~km}$ (Teanby et al. 2012; Vinatier et al. 2015), showing that the southern polar subsidence had already settled at that date. From the combined interpretation of the meridional cross sections of temperature and haze, $\mathrm{C}_{2} \mathrm{H}_{2}$, $\mathrm{HCN}$, and $\mathrm{C}_{2} \mathrm{H}_{6}$ volume mixing ratios (VMRs) derived from CIRS limb observations acquired between July $2009\left(L_{s}=359^{\circ}\right)$ and May $2013\left(L_{s}=45^{\circ}\right)$, Vinatier et al. (2015) showed that the global dynamics had entirely reversed within about two years after the northern spring equinox.

One of the most striking events of the southern fall was the unexpected $30 \mathrm{~K}$ cooling of the polar stratopause and mesosphere between June 2011 and February $2012\left(L_{s}=22.7^{\circ}-30.6^{\circ}\right)$ (Teanby et al. 2017; Vinatier et al. 2015) at least partly explained by the increased efficiency of radiative cooling by the strongly enhanced photochemical species and the decreasing solar flux, while the subsidence was strengthening the adiabatic heating (Teanby et al. 2017). The southern polar enrichment continued to strengthen after this date, reaching its maximum in March $2015\left(L_{s}=65.8^{\circ}\right.$; Mathé et al. 2020), while the polar stratospheric temperature continually decreased before increasing again around September $2015\left(L_{s}=72^{\circ}\right)$ (Teanby et al. 2017). The southern molecular polar enrichment combined with the low stratospheric temperatures were at the origin of the development of a large stratospheric polar cloud first observed in May 2012 by the Cassini Imager SubSystem (ISS; West et al. 2016). Several ice signatures were detected inside this cloud at different altitudes: HCN ice (de Kok et al. 2014; Le Mouélic et al. 2018), $\mathrm{C}_{6} \mathrm{H}_{6}$ ice (Vinatier et al. 2018), the "haystack" cloud at $220 \mathrm{~cm}^{-1}$ (Jennings et al. 2012, 2015) and the High Altitude South Polar (HASP) cloud possibly containing co-condensed $\mathrm{C}_{6} \mathrm{H}_{6}: \mathrm{HCN}$ ice (Anderson et al. 2018). Seasonal evolution of this cloud was monitored using the Cassini Visible and Infrared Mapping Spectrometer (VIMS) by Le Mouélic et al. (2018).

At high-northern latitudes, Teanby et al. (2019) and Coustenis et al. (2020) showed from analysis of CIRS observations acquired in nadir geometry that the northern winter photochemical species enhancements resulting from their transport by subsidence remained during the entire northern spring. They assumed constant-with-height VMR as vertical information is poor for most of molecules with this observing mode, which mostly probe the 1-10 mbar range. We show in our study that molecular VMR vertical profiles in this region varied greatly with altitude and that the northern spring polar enrichment was in fact confined to the mid-stratosphere $(0.1-1$ mbar pressure range).

Titan's deep stratosphere, at 15 mbar, also underwent seasonal variations at both poles with a temperature decrease of $25 \mathrm{~K}$ at $70^{\circ} \mathrm{S}$ between January 2007 and June 2016 and a temperature increase of $7 \mathrm{~K}$ at the north pole in the same period (Sylvestre et al. 2020). The south pole enriched air was also observed at the 15 mbar level at latitudes higher than $70^{\circ} \mathrm{S}$ in late 2012 (Sylvestre et al. 2018) with continuous increase of the VMR with time. Indeed, near $70^{\circ} \mathrm{S}, \mathrm{VMR}$ of $\mathrm{C}_{4} \mathrm{H}_{2}$ and $\mathrm{C}_{2} \mathrm{~N}_{2}$ were 30 and 40 times higher in 2016 than in 2009, respectively, while $\mathrm{CH}_{3} \mathrm{C}_{2} \mathrm{H}$ was enriched by a factor of 10 in the same period.

Our goal in this paper is to study in detail the seasonal evolution of the thermal field and the VMR meridional cross sections of nine molecules and aerosols, from one pole to another and from 5 mbar up to $50 \mu$ bar $(120-650 \mathrm{~km})$, to determine how the remaining northern polar enrichment resulting from the previous winter vanished at the approach of the northern summer solstice and how the southern polar vortex evolved during the second part of the southern fall. We aim to interpret these observations in terms of seasonal changes of the dynamics through comparison with prediction of the Institut Pierre Simon Laplace Titan Global Climate Model (IPSL Titan GCM).

We have analyzed CIRS mid-infrared spectra acquired at the limb of Titan from January 2015 to September 2017 (the end of the Cassini mission) to retrieve temperatures; VMRs of $\mathrm{C}_{2} \mathrm{H}_{2}$, $\mathrm{C}_{2} \mathrm{H}_{4}, \mathrm{C}_{2} \mathrm{H}_{6}, \mathrm{CH}_{3} \mathrm{C}_{2} \mathrm{H}, \mathrm{C}_{3} \mathrm{H}_{8}, \mathrm{C}_{4} \mathrm{H}_{2}, \mathrm{C}_{6} \mathrm{H}_{6}, \mathrm{HCN}$, and $\mathrm{HC}_{3} \mathrm{~N}$; haze extinction; and derived zonal wind in the stratosphere and the mesosphere. We have extended as much as possible our retrievals to provide new hints on dynamical and chemical processes occurring in the mesosphere. This study extends the work of Vinatier et al. (2015), which focused on the temperature field and the $\mathrm{C}_{2} \mathrm{H}_{2}, \mathrm{HCN}, \mathrm{C}_{2} \mathrm{H}_{6}$, and aerosol VMRs around the northern spring equinox, from July 2009 to May 2013, in the 5$10^{-3}$ mbar (120-500 km). Between 2012 and 2015, the Cassini orbit inclination was too high to probe both poles in limb geometry viewing, and it is why we focus here on limb data analysis between 2015 and the end of the Cassini mission in September 2017.

Section 2 describes the CIRS limb observations sequences used in this study. The retrieval method and the inferred temperature field and photochemical species VMR cross sections are presented in Sects. 3 and 4, respectively. Results are discussed in Sect. 5.

\section{Observations}

Our study is based on the analysis of thermal emission of Titan's limb acquired by the Cassini/CIRS spectrometer. CIRS was a Fourier transform spectrometer acquiring spectra in the farinfrared (from 10 to $600 \mathrm{~cm}^{-1}$ ) with a 3.9-mrad FWHM circular detector on Focal Plane 1 (FP1) and in the mid-infrared through two linear arrays each composed of ten 0.273-mrad field-of-view detectors on Focal Plane 3 (FP3, from 570 to $1125 \mathrm{~cm}^{-1}$ ) and Focal Plane 4 (FP4, from 1050 to $1495 \mathrm{~cm}^{-1}$ ). A detailed description of the CIRS instrument and an overview of the different observing modes are given in Kunde et al. (1996), Flasar et al. (2004) and Nixon et al. (2019). FP3 and FP4 were specially designed to probe the vertical structure of Titan's atmosphere, while these linear detector arrays were positioned parallel to a Titan radius when Cassini was at a distance from Titan's surface of 100 000-200 $000 \mathrm{~km}$, so that each detector had a vertical resolution of about one scale height $(\sim 40 \mathrm{~km})$. During a single limb sequence (above a given latitude and longitude), each FP3 and FP4 detector array pointed successively at two positions in the atmosphere: the deep atmosphere with the ten-detector line-ofsight altitudes spanning from the surface to about $350 \mathrm{~km}$ and then the upper stratosphere and mesosphere with lines of sight spanning the $\sim 300-700 \mathrm{~km}$ region. Each FP3 and FP4 detector 
array therefore pointed successively at two altitude ranges with an overlap of about $\sim 70 \mathrm{~km}$ in the $300-400 \mathrm{~km}$ altitude region. Combining the two pointing positions of FP3 and FP4 arrays allowed us to probe the atmosphere between 100 to $650 \mathrm{~km}$. As 30 to 50 spectra were usually acquired during a single position by each of the ten detectors of FP3 and FP4 at very similar altitudes, we averaged them per detector (and therefore for a given altitude level) to increase the signal-to-noise ratio $(\mathrm{S} / \mathrm{N})$ of the spectra used in our retrievals. In order to derive the temperature and photochemical compound global spatial and vertical distributions, we used dedicated FP3 and FP4 CIRS limb observation sequences, in which an entire hemisphere was scanned while Cassini was approaching or leaving Titan during a given flyby. Such observations had an apodized spectral resolution of $15.5 \mathrm{~cm}^{-1}$. More details on this observation type, called midinfrared limb maps (MIRLMBMAP), are given in Nixon et al. (2019).

Data used here are extracted from the "global calibration" version (Jennings et al. 2017) archived in the NASA Planetary Data System. We analyzed 11 MIRLMBMAP sequences and combined those acquired within two consecutive Titan flybys, usually within a two-month time period, to derive pole-to-pole spatial distributions of the temperature and photochemical compound VMRs. When these sequences did not cover the polar regions, we also analyzed spectra acquired in a nadir viewing geometry mode at $3-\mathrm{cm}^{-1}$ spectral resolution to complete the spatial distributions derived from limb observations. We then determined global spatial distributions for seven time slots from March 2015 to September 2017. Details of the observations used here are given in Tables B.1 and B.2.

\section{Retrieval of the temperature and photochemical compound mixing ratio profiles}

The observed molecular rovibrational emission bands intensities depend on both temperature and molecular VMRs. The continuum emission is due to the haze extinction and the collisioninduced absorption of $\mathrm{N}_{2}-\mathrm{N}_{2}, \mathrm{~N}_{2}-\mathrm{CH}_{4}, \mathrm{CH}_{4}-\mathrm{CH}_{4}$, and $\mathrm{N}_{2}-\mathrm{H}_{2}$. In order to infer both temperature and chemical compound VMR vertical profiles, we proceeded in several steps. In the first step we retrieved simultaneously, from the FP4 limb spectra, the temperature profile by fitting the $v_{4}-\mathrm{CH}_{4}$ band in the 1200 $1330 \mathrm{~cm}^{-1}$ spectral range and the haze extinction profile from the fit of the continuum in the $1080-1120 \mathrm{~cm}^{-1}$ spectral range. In the second step these profiles were incorporated in our atmospheric model to derive the molecular VMR profiles from the fit of their emission bands in FP3 limb spectra. For both steps we determined the vertical profiles using an inversion algorithm based on the fit of the observed limb spectra using a line-by-line radiative transfer code. The inversion algorithm and the spectroscopic files used here are described in Vinatier et al. (2010b, 2015). We additionally utilized here the propane pseudo-line list of Sung et al. (2013) to reproduce the $\mathrm{C}_{3} \mathrm{H}_{8} v_{7}, v_{21}, v_{20}$, and $v_{7}$ emission bands centered at $869,922,1054$, and $1158 \mathrm{~cm}^{-1}$, respectively. We used the spectral dependence of the aerosol extinction coefficient from Vinatier et al. (2012) to reproduce the continuum of all selected limb spectra. As in our previous studies (Vinatier et al. 2007, 2010a, 2015), we had to apply a shift on the CIRS database nominal line-of-sight altitudes of our selected limb spectra to reproduce the P- and Q-branches of the $v_{4}-\mathrm{CH}_{4}$ band of the deepest limb spectra, which display the highest $\mathrm{S} / \mathrm{Ns}$. In previous analyses we applied this shift to the lines of sight of all averaged limb spectra acquired in a given limb; at the beginning of the Cassini mission the relative pointing of the two positions of the FP4 detector array in a given limb sequence was accurate so that radiances of detectors in the overlapping altitude region were consistent within the Noise Equivalent Spectral Radiance (Teanby et al. 2007). However, for the limb sequences used here, we found a discontinuity in the vertical profile of the limb integrated radiance in the overlapping zone of the two pointing positions of the FP4 array. To ensure a continuity of the integrated radiance profile, we first determined the shift to apply to the lowest pointing position where limb spectra have the highest S/Ns. After applying this shift to the lines of sight of the deepest position of the FP4 array, we then determined what shift to apply to the pointing altitudes of the upper pointing position to get a continuity of the entire radiance vertical profile (including both pointing positions). A similar method was also used by Mathé et al. (2020) for all CIRS limb spectra acquired at a $0.5-\mathrm{cm}^{-1}$ spectral resolution. The applied shifts are given in Table B.1. We applied the same shifts to the limb spectra acquired by the FP3 because FP4 and FP3 spectra were acquired simultaneously.

After constraining the temperature profile, we incorporated it in our atmospheric model to reproduce the radiance of the molecular emission bands in the FP3 spectral region. At $15.5 \mathrm{~cm}^{-1}$ resolution, several molecular emission bands are mixed (i.e., for $\mathrm{C}_{4} \mathrm{H}_{2}$ and $\mathrm{CH}_{3} \mathrm{C}_{2} \mathrm{H}$ or $\mathrm{HC}_{3} \mathrm{~N}, \mathrm{CO}_{2}$, and $\mathrm{C}_{6} \mathrm{H}_{6}$; see Fig. 1). The only well-separated emission bands are those of $\mathrm{C}_{2} \mathrm{H}_{6}$ and $\mathrm{C}_{2} \mathrm{H}_{4}$. The haze thermal emission also contributes to the continuum in the entire FP3 spectral range. In order to properly reproduce the continuum, we retrieved the haze optical depth profile simultaneously with $\mathrm{C}_{4} \mathrm{H}_{2}$ and $\mathrm{CH}_{3} \mathrm{C}_{2} \mathrm{H}$ mixing ratio profiles from the $595-660 \mathrm{~cm}^{-1}$ spectral range in which the $595-610 \mathrm{~cm}^{-1}$ region is free of molecular emission bands. We then utilized this retrieved haze optical depth profile to model the continuum radiance in the entire $\mathrm{FP} 3$ spectral range.

As $\mathrm{HC}_{3} \mathrm{~N}, \mathrm{CO}_{2}$, and $\mathrm{C}_{6} \mathrm{H}_{6}$ emission bands are mixed, and as the $\mathrm{CO}_{2} \mathrm{VMR}$ does not vary with latitude or season (Vinatier et al. 2015; Mathé et al. 2020), we fixed the $\mathrm{CO}_{2}$ VMR profile to the one derived by Vinatier et al. (2015) at $0^{\circ} \mathrm{N}$ in May 2012. The $\mathrm{HC}_{3} \mathrm{~N}$ and $\mathrm{C}_{6} \mathrm{H}_{6}$ mixing ratio profiles were retrieved from the fit of the $660-678 \mathrm{~cm}^{-1}$ spectral range. $\mathrm{C}_{2} \mathrm{H}_{2}, \mathrm{HCN}$, and $\mathrm{C}_{3} \mathrm{H}_{8}$ were retrieved simultaneously from the $705-750 \mathrm{~cm}^{-1}$ range, $\mathrm{C}_{2} \mathrm{H}_{6}$ was retrieved from the $800-850 \mathrm{~cm}^{-1}$ range, and $\mathrm{C}_{2} \mathrm{H}_{4}$ from the $900-986 \mathrm{~cm}^{-1}$ region (excluding the $952.5-958 \mathrm{~cm}^{-1}$ region because of an instrumental noise spike there). Examples of fits of these spectral ranges are displayed in Fig. 1.

Even though the detected emission in the mesosphere cannot entirely be considered in local thermodynamic equilibrium (LTE; Feofilov et al. 2016), we chose to retrieve thermal and VMR profiles at altitudes as high as possible, assuming LTE everywhere in order to highlight upper levels in which information is available, postponing a more appropriate retrieval including non-LTE effects for a later dedicated study.

\section{Results}

\subsection{Temperature field}

The temperature meridional cross sections retrieved from $L_{s}=66^{\circ}$ (March 2015) to $L_{s}=93^{\circ}$ (September 2017) are displayed in Fig. 2 (with a pressure $y$-axis) and in Fig. C.1 (polar representation with an altitude vertical axis calculated assuming hydrostatic equilibrium). The polar cross section of March-May 2015 is the only one that extends across both poles (see Fig. C.1). In both figures, regions without information (in white) correspond either to a lack of data, poor S/Ns, or altitude levels not 

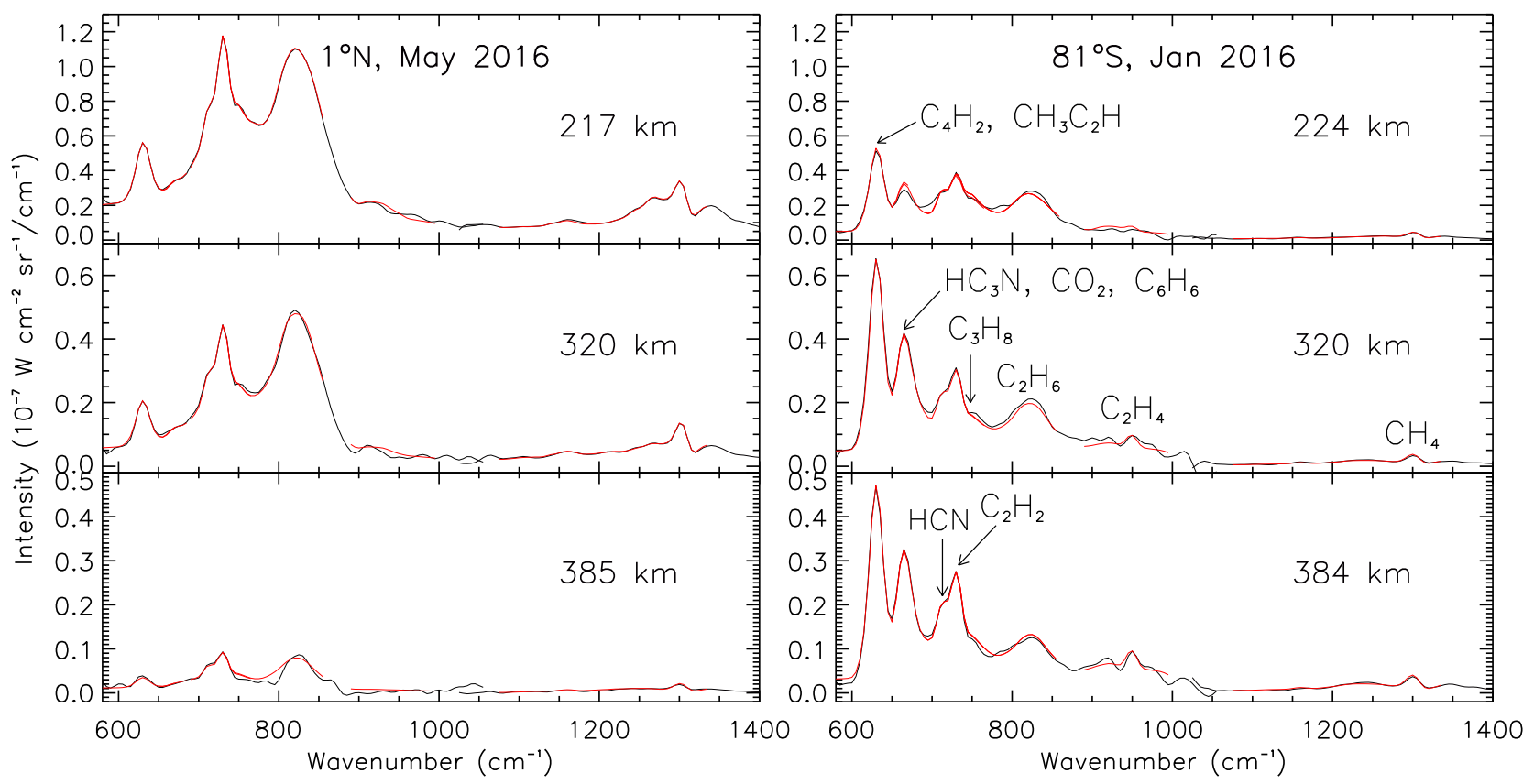

Fig. 1. Example of CIRS limb spectra acquired at $1^{\circ} \mathrm{N}$ in July 2016 (left panel, black line) and $81^{\circ} \mathrm{S}$ in January 2016 (right panel, black line) at three different tangent heights. They are compared with the best fit spectra (in red). These spectra can be compared with those of Fig. 1 of Mathé et al. (2020, their Figs. 1 and 2) acquired at similar location, altitude, and date at higher spectral resolution. The band centered at $630 \mathrm{~cm}^{-1}$ includes $\mathrm{C}_{4} \mathrm{H}_{2}$ and $\mathrm{CH}_{3} \mathrm{C}_{2} \mathrm{H}$ emission; the band centered at $673 \mathrm{~cm}^{-1}$ includes $\mathrm{HC}_{3} \mathrm{~N}, \mathrm{CO}_{2}$, and $\mathrm{C}_{6} \mathrm{H}_{6}$. $\mathrm{HCN}, \mathrm{C}_{2} \mathrm{H}_{2}$, and $\mathrm{C}_{3} \mathrm{H}_{8}$ emission bands are centered respectively at 713,730 , and $748 \mathrm{~cm}^{-1} . \mathrm{C}_{2} \mathrm{H}_{6}, \mathrm{C}_{2} \mathrm{H}_{4}$, and $\mathrm{CH}_{4}$ bands are centered at 815,950 , and $1300 \mathrm{~cm}^{-1}$, respectively. An overlap between FP3 and FP4 spectra occurs in the $1000-1050 \mathrm{~cm}^{-1}$ spectral range.

probed by nadir observations. Temperature profiles derived from limb observations were usually constrained in the $120-650 \mathrm{~km}$ range $\left(\sim 5\right.$ mbar to $5 \times 10^{-5}$ mbar $)$, while nadir observations probed the $120-400 \mathrm{~km}$ region ( $\sim 5 \mathrm{mbar}$ to $\left.10^{-2} \mathrm{mbar}\right)$ except at high-southern latitudes where they probed up to $550 \mathrm{~km}$ $\left(\sim 5 \times 10^{-4}\right.$ mbar) because of the very steep vertical thermal gradient there. The retrieved thermal profiles including their error bars are available in supplementary material and will be posted on the Virtual European Solar and Planetary Access (VESPA) portal $^{1}$. Error bars are usually smaller than $1 \mathrm{~K}$ below the stratopause and can reach a few kelvins at mesospheric levels. Error bars include uncertainties due to spectral noise and the $\pm 2 \mathrm{~km}$ uncertainty on the determined vertical shift.

\subsubsection{High-southern latitudes}

The most striking seasonal changes in temperature between $L_{s}=66^{\circ}$ and $L_{s}=93^{\circ}$ were observed in the southern hemisphere at latitudes higher than $30^{\circ} \mathrm{S}$, where the stratopause altitude varied with latitude in a complex way. The southern polar stratopause reached its highest temperature close to the south pole (Figs. 2 and C.1), its temperature increased by $10 \mathrm{~K}$ from March to September $2015\left(L_{s}=66^{\circ}-72^{\circ}\right)$ and by $22 \mathrm{~K}$ from May 2016 to February $2017\left(L_{s}=78^{\circ}-87^{\circ}\right)$ to reach its highest value of $207 \mathrm{~K}$. For the time period studied here, the altitude of the stratopause was globally decreasing towards mid-latitudes.

At deeper levels, the polar stratosphere was at its coldest in March and September $2015\left(L_{s}=66^{\circ}\right.$ and $\left.L_{s}=72^{\circ}\right)$ with temperatures lower than $120 \mathrm{~K}$ observed below the $0.06 \mathrm{mbar}$ pressure level $(260 \mathrm{~km}$ altitude) in March and the $0.2 \mathrm{mbar}$

\footnotetext{
1 Vertical profiles are accessible on the VESPA portal: (http:// vespa.obspm.fr/planetary/data/). Fits of the observed spectra are available on request to $S$. Vinatier.
}

level $(\sim 200 \mathrm{~km})$ in September. As the southern autumn was progressing, these low temperatures were observed to propagate deeper.

\subsubsection{Mid-southern latitudes}

Interestingly, the $30^{\circ} \mathrm{S}$ latitude seemed to be a transition in the structure of the thermal field from September $2015\left(L_{s}=72^{\circ}\right)$ to May $2016\left(L_{s}=78^{\circ}\right)$ : on the low-latitude side, the stratopause was located deeper $(\sim 0.2 \mathrm{mbar}, 250 \mathrm{~km})$ than toward higher latitudes (at $40^{\circ} \mathrm{S}$, it was located at $3 \times 10^{-3} \mathrm{mbar}, 450 \mathrm{~km}$ ). It resulted in a double $173 \mathrm{~K}$ stratopause structure at $30^{\circ} \mathrm{S}$ (Figs. 2 and C.1). On both sides of $30^{\circ} \mathrm{S}$, the stratopause remained at similar pressure levels until May 2016, while in February 2017 the two local temperature maxima seemed to have merged into a single one around $3 \times 10^{-2} \mathrm{mbar}(340 \mathrm{~km})$. This new structure was observed until the southern winter solstice in May 2017, the last date for which we have limb observations at this latitude.

\subsubsection{Northern hemisphere}

Thermal profiles in the northern hemisphere showed moderate changes during the second half of the northern spring. Altitude of the stratopause remained the same $(\sim 300 \mathrm{~km}, 0.1 \mathrm{mbar})$ at all latitudes from $L_{s}=67^{\circ}$ to $L_{s}=93^{\circ}$ (May 2015 to September 2017) with similar temperature (about 175-180 K) everywhere except at latitudes higher than $70^{\circ} \mathrm{N}$, where the stratopause was typically $5 \mathrm{~K}$ warmer than at lower latitudes. After the northern summer solstice (for $L_{s}=93^{\circ}$ ), at high-northern latitudes the stratosphere was $\sim 2 \mathrm{~K}$ colder and the stratopause $5 \mathrm{~K}$ colder than at $L_{s}=76^{\circ}$ (February 2016), while an opposite trend was observed in the mesosphere, which was $\sim 5 \mathrm{~K}$ warmer (in the $0.2-1 \times 10^{-2}$ mbar region) after the northern summer solstice than earlier in the spring. 
S. Vinatier et al.: Seasonal variations in Titan's atmosphere close to the northern summer solstice
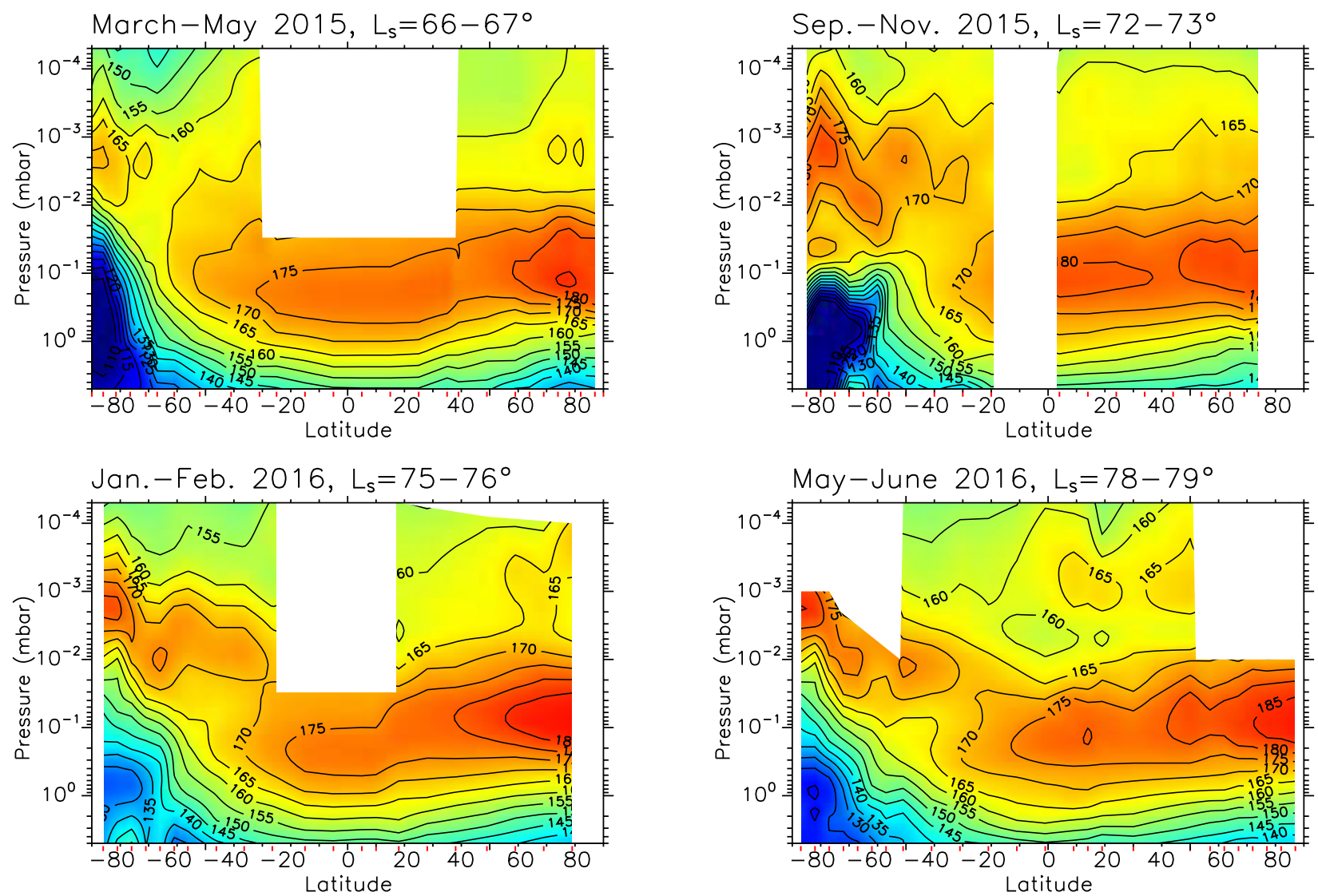

Feb. $2017, L_{s}=87^{\circ}$
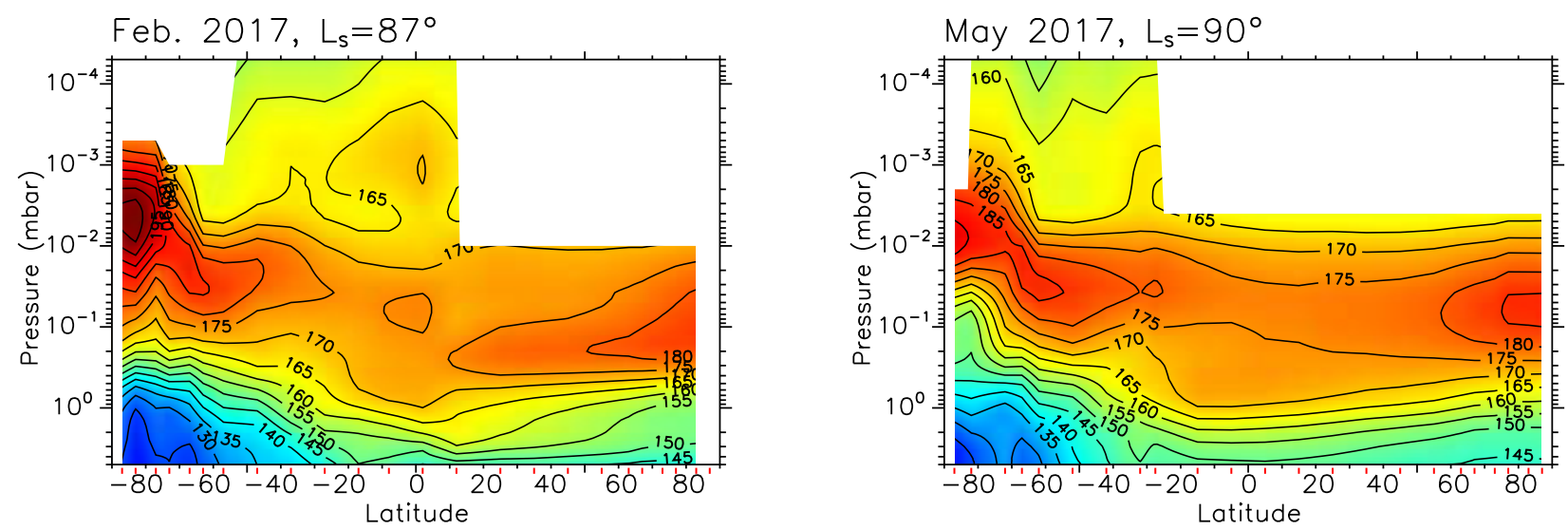

Sept. $2017, L_{s}=93^{\circ}$
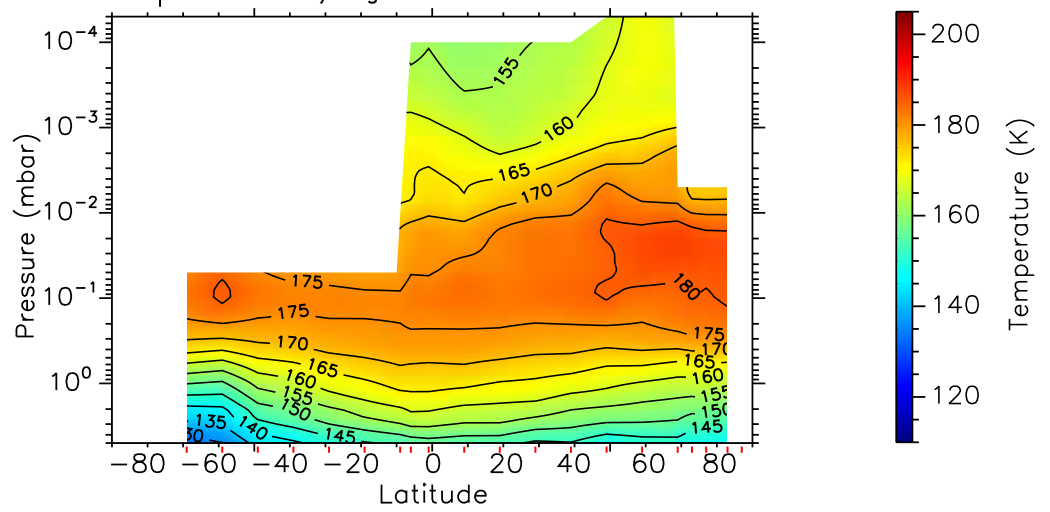

Fig. 2. Temperature meridional cross sections from March 2015 to September 2017. Contours are given every $5 \mathrm{~K}$ from 110 to $205 \mathrm{~K}$; the color scale is the same for all plots. In the equatorial region, pressures of $1,0.1,0.01,10^{-3}$, and $10^{-4}$ mbar roughly correspond to altitudes of 200,300 , 400,500 , and $600 \mathrm{~km}$, respectively. Regions without information are represented in white; they correspond to missing data or pressure levels not probed by nadir observations. Red ticks on the $x$-axis give latitudes of observations used to retrieved the global map. 


\subsubsection{Possible correlation between mesospheric temperature and insolation}

We infer a local temperature maximum in the mesosphere around $500 \mathrm{~km}\left(10^{-3}\right.$ mbar $)$ during Titan's daytime. For instance, a $165 \mathrm{~K}$ temperature local maximum was observed at this pressure level at high-northern latitudes in May 2015, at a solar zenith angle (SZA) of $63-90^{\circ}$, and February 2016 (17:00 local time, $\left.\mathrm{SZA}=60-66^{\circ}\right)$, at mid-northern latitudes and the equator in June $2016\left(\sim 15: 00\right.$ local time, $\left.\mathrm{SZA}=30-60^{\circ}\right)$, and around the equator and at low-southern latitudes in February 2017 (12:30 local time, $\left.\mathrm{SZA}=19-45^{\circ}\right)$. In contrast, it seems that the coldest mesospheric temperatures were observed during the night; for instance, temperatures about $5 \mathrm{~K}$ higher were found at the end of the day above $10^{-3}$ mbar at mid-northern latitudes in November 2015 (local time $\sim 17: 50, \mathrm{SZA}=68-85^{\circ}$ ) than in the middle of the night in May 2015 (local time $\sim 01: 30$, SZA of $95-113^{\circ}$ ). In the southern hemisphere the lowest mesospheric temperatures were also observed during the night; for instance, in February 2017, the $10^{-4}$-mbar level temperature was found to increase from $162 \mathrm{~K}$ at $09: 22$ local time at $50^{\circ} \mathrm{S}\left(\mathrm{SZA}=82^{\circ}\right)$ to $167 \mathrm{~K}$ at $12: 48$ at $2^{\circ} \mathrm{N}$ $\left(\mathrm{SZA}=26^{\circ}\right)$.

In the northern hemisphere, which was experiencing spring, we also inferred that the more illuminated high-northern latitudes showed higher mesospheric temperatures. For instance, in September 2017 the mid-northern latitude mesosphere (from Equator to $40^{\circ} \mathrm{N}, \mathrm{SZA}=106-153^{\circ}, 21: 30-22: 47$ local time) was $\sim 5 \mathrm{~K}$ colder at $10^{-4}$ mbar than the constantly insolated highnorthern latitude (local time $\sim 20: 30, \mathrm{SZA}=72-85^{\circ}$ ). A similar mesospheric temperature trend was also observed in May 2015 as the colder mid-northern latitude mesosphere was in the night $\left(\sim 01: 30\right.$ local time, $\left.\mathrm{SZA}=90-113^{\circ}\right)$ and the high-northern latitude mesosphere was insolated $\left(\mathrm{SZA}=60-86^{\circ}\right)$.

Mesospheric temperatures seem to be correlated with solar insolation even if adiabatic heating (respectively cooling) due to air subsidence (respectively upwelling) can also impact the mesospheric temperatures. This possible correlation, which can only be detectable in the mesosphere where the radiative time constant is about a few Titan hours (Bézard et al. 2018), was not observed deeper at the stratopause and stratospheric levels, consistently with the predicted radiative time constant greater than one Titan day there (Bézard et al. 2018).

\subsection{Molecular gas mixing ratio meridional cross sections}

Outside the southern polar region $\mathrm{C}_{2} \mathrm{H}_{2}, \mathrm{HCN}$ (Figs. 3, A.2, and C.1 middle and bottom panel) and $\mathrm{C}_{2} \mathrm{H}_{6}$ (Figs. A.1 and C.2 bottom panel) are the molecules whose VMR could be derived up to a few $10^{-4}$ mbar (500-600 km). $\mathrm{C}_{4} \mathrm{H}_{2}$ and $\mathrm{CH}_{3} \mathrm{C}_{2} \mathrm{H} \mathrm{VMR}$ (Figs. A.3, A.4 and C.2) were generally determined up to a few $10^{-3}$ mbar (450-500 km). $\mathrm{C}_{3} \mathrm{H}_{8}$ and $\mathrm{C}_{2} \mathrm{H}_{4}$ mixing ratios (Figs. A.5, A.8, C.3 and C.4) were usually inferred up to $10^{-2}$ $10^{-3}$ mbar $\left(400-450 \mathrm{~km}\right.$ ) and $\mathrm{HC}_{3} \mathrm{~N}$ and $\mathrm{C}_{6} \mathrm{H}_{6}$ (Figs. A.6, A.7, and C.3) were mostly detected at high latitudes up to $600 \mathrm{~km}$ at the south pole, while in the mid- and low-latitude regions, their VMRs (usually lower than $10^{-9}$ ) should be considered as upper limits. Retrieved mixing ratio profiles including their error bars are available in the supplementary material and will be posted on the Virtual European Solar and Planetary Access (VESPA) portal $^{2}$. Determination of the error bars follows the methodology described by Vinatier et al. (2010a). They include the uncertainty

\footnotetext{
2 Vertical profiles are accessible on the VESPA portal: http:// vespa.obspm.fr/planetary/data/). Fits of the observed spectra are available on demand to $S$. Vinatier.
}

on the retrieved thermal profiles, the $\pm 2 \mathrm{~km}$ uncertainty on the vertical shift, and the spectral noise, all summed quadratically at each pressure level.

Before looking in detail at the spatial distributions of each molecule, we first depict the main common global spatial and temporal trends of the inferred VMR:

- Most molecules were strongly enriched at high-southern latitudes inside the polar vortex because of the subsidence of the global circulation cell that settled in 2012 (Teanby et al. 2012; Vinatier et al. 2015) and transported enriched air from higher levels toward the deep stratosphere. Additionally, this polar air was isolated from the air at mid-southern latitudes by strong zonal winds (Achterberg et al. 2011, 2018; West et al. 2016) forming the polar vortex (see also Sect. 5.5). At a given date, all polar VMR distributions (except those of $\mathrm{C}_{2} \mathrm{H}_{6}$ and $\mathrm{C}_{3} \mathrm{H}_{8}$ that have limited vertical gradients and therefore show limited enhancement by subsidence) showed very steep meridional gradients at high-southern latitudes, tracing the polar vortex barrier (Teanby et al. 2008). Depending on the molecules, polar enhancements were the greatest in 2015 and in January 2016. In March 2015 the molecules showing the steepest concentration meridional gradients across the polar vortex boundary were $\mathrm{C}_{2} \mathrm{H}_{4}, \mathrm{C}_{3} \mathrm{H}_{4}, \mathrm{C}_{6} \mathrm{H}_{6}$, $\mathrm{HC}_{3} \mathrm{~N}$, and $\mathrm{HCN}$, which varied from a factor of 10 for $\mathrm{HCN}$ to three orders of magnitude for $\mathrm{HC}_{3} \mathrm{~N}$ and $\mathrm{C}_{6} \mathrm{H}_{6}$. In September 2015, this polar enriched region had a wider latitudinal extension than in March 2015 and January 2016, and displayed stronger enrichments than in May 2017, suggesting changes in the horizontal structure of the polar vortex over this period.

- In May 2017, at high-southern latitudes (between $30^{\circ} \mathrm{S}$ and $\left.60^{\circ} \mathrm{S}\right)$ and at altitudes higher than $500 \mathrm{~km}\left(10^{-3} \mathrm{mbar}\right)$, $\mathrm{C}_{2} \mathrm{H}_{2}, \mathrm{C}_{2} \mathrm{H}_{6}, \mathrm{C}_{4} \mathrm{H}_{2}, \mathrm{CH}_{3} \mathrm{C}_{2} \mathrm{H}$, and $\mathrm{HCN}$ were more abundant than earlier in the season, while their polar enhancements in the $10^{-1}-10^{-3}$ mbar region were less pronounced, suggesting some changes in the global circulation occurring close to the southern winter solstice.

- In the deep stratosphere, typically below the 1 mbar level, at mid- and low latitudes all molecular VMRs (except for $\mathrm{C}_{2} \mathrm{H}_{4}$ which does not condense) decreased with depth as the main sink of these molecules is condensation, usually occurring below the 10 mbar level $(\sim 100 \mathrm{~km})$. Some molecules, like $\mathrm{HCN}, \mathrm{C}_{4} \mathrm{H}_{2}$, and $\mathrm{CH}_{3} \mathrm{C}_{2} \mathrm{H}$, show steep slopes in the contours of constant mixing ratio at low- or mid-latitudes (see, e.g., Fig. A.4 in May-June 2016 or Fig. A.2 in Sept.-Nov. 2015), which suggests that upwelling was transporting deeper air (depleted due to condensation) to upper levels in the stratosphere.

- Mesospheric depletions in $\mathrm{C}_{2} \mathrm{H}_{2}, \mathrm{HCN}$, and $\mathrm{C}_{2} \mathrm{H}_{6}$ (Figs. 3, A.1, A.2, C.1 and C.2), which are the molecules for which we can derive information at the highest levels, have been observed since September-November $2015\left(L_{s}=72-73^{\circ}\right)$. This depletion seems to have been initiated at high-northern latitudes in May $2015\left(L_{s}=67^{\circ}\right)$ and has extended down to mid-southern latitudes in September $2015\left(L_{s}=72^{\circ}\right)$.

- Although at high-northern latitudes the upwelling branch of the global circulation cell has been in place since probably mid-2011 (Vinatier et al. 2015), a region located at latitudes higher than $60^{\circ} \mathrm{N}$ and altitudes between 200 and $300 \mathrm{~km}$ (Figs. 3 to 4 and Figs. C.1 to C.4) remained enriched in photochemical compounds (including haze) until the northern summer solstice, with a tendency to vanish after this date. Most of these molecular enrichments were located at the stratopause level. This suggests that the stratopause could be a sort of stagnancy region with limited mixing or that the northern winter residual circulation cell (which keeps some of the winter polar enriched air in the deep stratosphere up to the stratopause) was still present over the 
S. Vinatier et al.: Seasonal variations in Titan's atmosphere close to the northern summer solstice
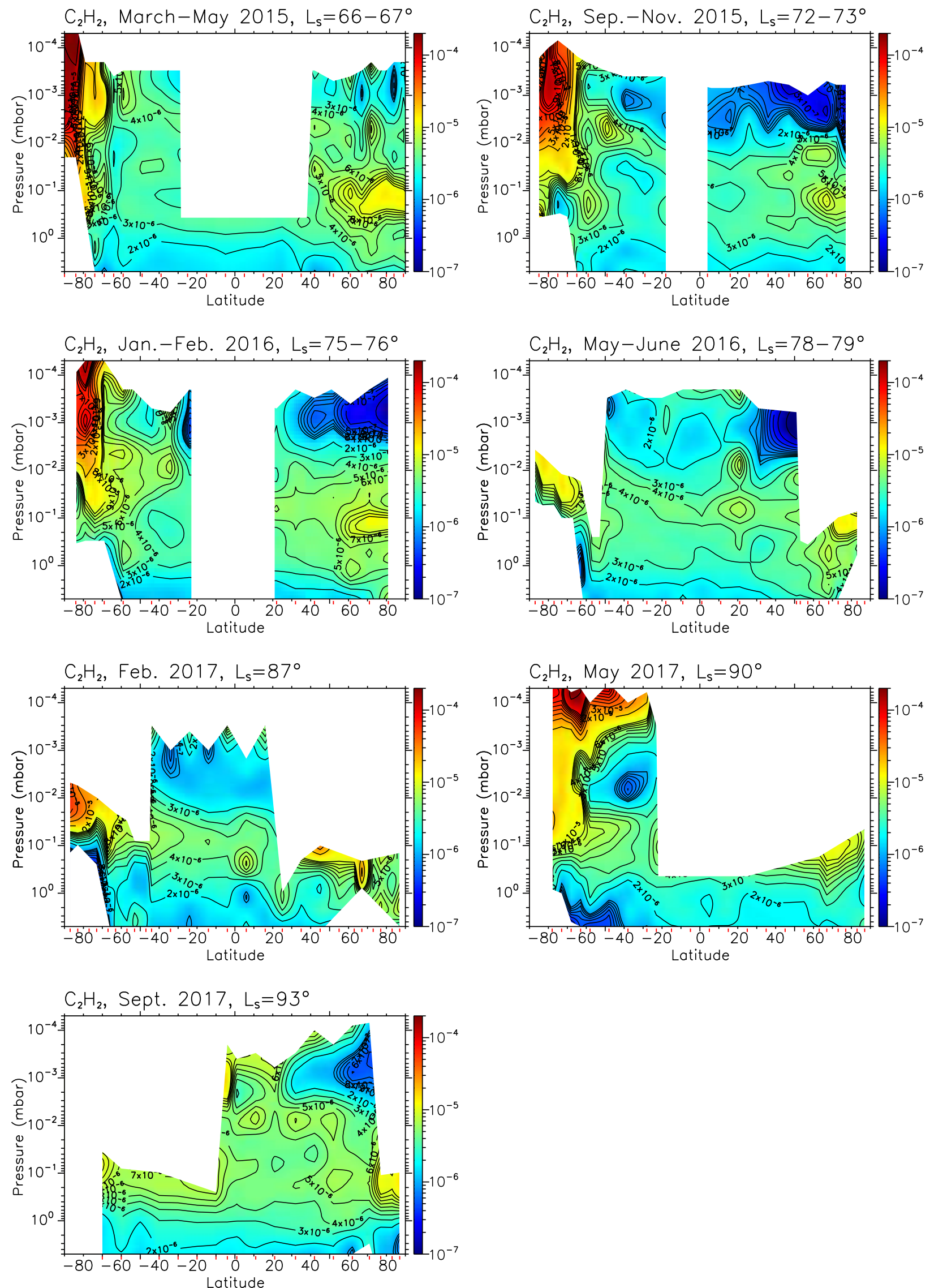

Fig. 3. $\mathrm{C}_{2} \mathrm{H}_{2}$ volume mixing ratio meridional cross sections from March 2015 to September 2017. Regions without information are represented in white; they correspond to missing data or pressure levels not probed by nadir observations. Red ticks on the $x$-axis give latitudes of observations used to retrieved the global map. 

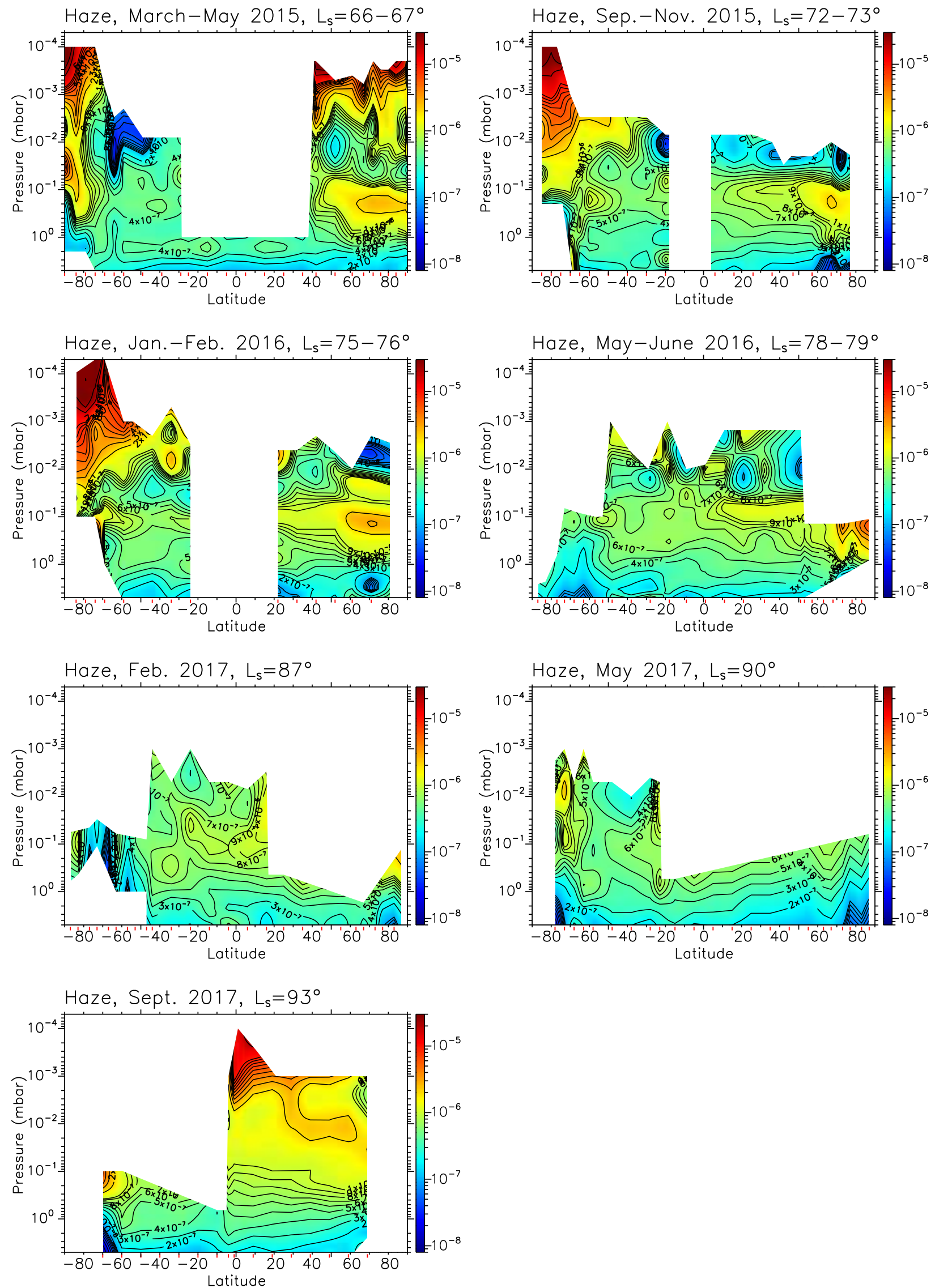

Fig. 4. Haze mass mixing ratio meridional cross sections from March 2015 to September 2017, determined from the haze extinction meridional cross sections displayed in Fig. A.9. Regions without information are represented in white; they correspond to missing data or pressure levels not probed by nadir observations. 
entire spring, as predicted by the IPSL Titan GCM, albeit limited to the deep stratosphere for all compounds (see Sect. 5.6).

In the following subsections we detail some specific trends observed for groups of molecules showing similar spatial and temporal behavior.

\subsection{1. $\mathrm{C}_{2} \mathrm{H}_{2}, \mathrm{HCN}, \mathrm{C}_{2} \mathrm{H}_{6}$, and $\mathrm{C}_{3} \mathrm{H}_{8}$ VMR meridional cross sections}

The most abundant trace gases are $\mathrm{C}_{2} \mathrm{H}_{6}, \mathrm{C}_{2} \mathrm{H}_{2}, \mathrm{HCN}$ and $\mathrm{C}_{3} \mathrm{H}_{8}$. Their mixing ratios (with the exception of $\mathrm{C}_{3} \mathrm{H}_{8}$ ) can be derived at the highest altitudes from one pole to another (typically up to $10^{-3}-10^{-4}$ mbar, 500-600 km).

The $\mathrm{C}_{2} \mathrm{H}_{6}$ and $\mathrm{C}_{3} \mathrm{H}_{8}$ VMRs (Figs. A.1, A.5, C.2 and C.3) show the less pronounced stratospheric vertical gradient of all the studied molecules. While $\mathrm{C}_{2} \mathrm{H}_{6}$ can be detected up to $550-650 \mathrm{~km}, \mathrm{C}_{3} \mathrm{H}_{8}$ is usually determined up to $400 \mathrm{~km}\left(10^{-3}\right.$ $10^{-4}$ mbar). Both molecular VMRs varied at most by a factor of 5 in the whole stratosphere and showed a factor of 3 southern polar enhancement compared to mid-southern latitudes in the stratosphere and mesosphere. At high-northern latitudes, between 150 and $250 \mathrm{~km}$ (1-0.1 mbar), $\mathrm{C}_{3} \mathrm{H}_{8}$ remained enriched by a factor of 4 compared to latitudes lower than $60^{\circ} \mathrm{N}$ from 2015 to September 2017 and $\mathrm{C}_{2} \mathrm{H}_{6}$ remained enriched by a factor of 2 from 2015 to May 2017, before being depleted after the northern summer solstice.

In the northern mesosphere, above the $10^{-2}$ mbar level (400 km), between May and November 2015, we derived strong depletions of $\mathrm{C}_{2} \mathrm{H}_{2}, \mathrm{HCN}$ (both depleted by a factor of $\sim 10$ at $10^{-3}$ mbar, $500 \mathrm{~km}$ altitude, Figs. 3 , A.2 and C.1), and $\mathrm{C}_{2} \mathrm{H}_{6}$ (depleted by a factor of $\sim 2$ ). This depletion was also observed at mid-southern latitudes at the same pressure level in September 2015, in 2016, and up to May 2017, while in September 2017 this depletion was only observed at latitudes higher than $50^{\circ} \mathrm{N}$.

In May 2017, in the southern mesosphere, $\mathrm{C}_{2} \mathrm{H}_{2}, \mathrm{HCN}$, and $\mathrm{C}_{2} \mathrm{H}_{6}$ were enriched above the $10^{-3}$ mbar level compared to earlier observations. Simultaneously, the southern polar stratosphere was less enriched in $\mathrm{C}_{2} \mathrm{H}_{2}$ and $\mathrm{HCN}$ than in 2015 and 2016, which suggests some global dynamical changes at high-southern latitudes near the southern winter solstice.

In the deep polar southern stratosphere, in 2015 and 2016, the combined low temperatures and high polar enhancements resulted in saturation of $\mathrm{HCN}$ occurring at unusually high altitudes: at 0.05 mbar $(250 \mathrm{~km})$ in March 2015 and $0.15 \mathrm{mbar}$ $(195 \mathrm{~km})$ in September 2015. This is consistent with the detection of HCN ice in the VIMS spectra of the southern polar stratospheric cloud since mid-2012 (de Kok et al. 2014; Le Mouélic et al. 2018).

Interestingly, in 2015 and 2016 the cross section contours of constant HCN VMR at low latitudes in the deep stratosphere showed steeper slopes than those of $\mathrm{C}_{2} \mathrm{H}_{2}, \mathrm{C}_{2} \mathrm{H}_{6}$, and $\mathrm{C}_{3} \mathrm{H}_{8}$; these three VMRs were more or less constant at a given pressure level for all the dates studied here. As the HCN VMR vertical gradient is steeper than those of $\mathrm{C}_{2} \mathrm{H}_{2}, \mathrm{C}_{2} \mathrm{H}_{6}$, and $\mathrm{C}_{3} \mathrm{H}_{8}$, vertical dynamical transport has a larger impact on the HCN VMR profile. The overall shape of the HCN VMR contours at mid- and low latitudes suggests stratospheric upwelling of air at mid- and low latitudes in 2015 and 2016.

\subsection{2. $\mathrm{C}_{4} \mathrm{H}_{2}$ and $\mathrm{CH}_{3} \mathrm{C}_{2} \mathrm{H}$ VMR meridional cross sections}

The molecules $\mathrm{C}_{4} \mathrm{H}_{2}$ and $\mathrm{CH}_{3} \mathrm{C}_{2} \mathrm{H}$ (Figs. A.3, A.4, and C.2) show quite comparable stratospheric and mesospheric VMRs, even though $\mathrm{CH}_{3} \mathrm{C}_{2} \mathrm{H}$ was typically $\sim 3$ times more abundant than $\mathrm{C}_{4} \mathrm{H}_{2}$ above the 0.5 mbar level, and the $\mathrm{C}_{4} \mathrm{H}_{2}$ VMR vertical gradient is steeper than that of $\mathrm{CH}_{3} \mathrm{C}_{2} \mathrm{H}$ in the deep stratosphere, between 1 and 5 mbar.

Like other molecules, $\mathrm{C}_{4} \mathrm{H}_{2}$ and $\mathrm{CH}_{3} \mathrm{C}_{2} \mathrm{H}$ were strongly enriched in the southern polar region compared with lower southern latitudes. For instance, in March 2015 at the $0.1 \mathrm{mbar}$ level $(\sim 250 \mathrm{~km})$ the $\mathrm{C}_{4} \mathrm{H}_{2}$ VMR increased from $10^{-8}$ at $69^{\circ} \mathrm{S}$ to $7 \times 10^{-7}$ at $84^{\circ} \mathrm{S}$ and $\mathrm{CH}_{3} \mathrm{C}_{2} \mathrm{H}$ varyied from $2 \times 10^{-8}$ to $2 \times 10^{-6}$ in the same latitude range. In the mesosphere, at $10^{-3} \mathrm{mbar}$, within the same latitude range, both molecules showed enhancement of a factor of 10 in 2015 and in January 2016, and a factor of 5 in May 2017. These enhancements were comparable to those observed for $\mathrm{C}_{2} \mathrm{H}_{2}$ and $\mathrm{HCN}$ at the same pressure level in the same period.

At high-northern latitudes, both molecules were locally enriched by a factor of 7 to 9 in a limited zone comprised between 1 and $0.1 \mathrm{mbar}$ and from $50^{\circ} \mathrm{N}$ to the pole. In September 2017, after the northern summer solstice, this enriched region seemed to have disappeared for $\mathrm{C}_{4} \mathrm{H}_{2}$ and was still observed for $\mathrm{CH}_{3} \mathrm{C}_{2} \mathrm{H}$. In contrast to $\mathrm{C}_{2} \mathrm{H}_{2}$ and $\mathrm{HCN}$, which showed a depleted layer at pressures lower than $10^{-2}$ mbar (as previously discussed), $\mathrm{C}_{4} \mathrm{H}_{2}$ and $\mathrm{CH}_{3} \mathrm{C}_{2} \mathrm{H}$ showed enhancements in this region.

\subsection{3. $\mathrm{C}_{2} \mathrm{H}_{4}, \mathrm{C}_{6} \mathrm{H}_{6}, \mathrm{HC}_{3} \mathrm{~N}$ VMR meridional cross sections}

$\mathrm{C}_{2} \mathrm{H}_{4}, \mathrm{HC}_{3} \mathrm{~N}$, and $\mathrm{C}_{6} \mathrm{H}_{6}$ (Figs. A.6, A.7, A.8, C.3, and C.4) are the molecules that showed the strongest meridional enhancements at high-southern latitudes from 2015 to 2017. For instance, in the $10^{-2}-10^{-3}$ mbar range these molecules were enriched between $70^{\circ} \mathrm{S}$ and $84^{\circ} \mathrm{S}$ by factors of 130,100 , and 40 , respectively, with respect to latitudes lower than $65^{\circ} \mathrm{S}$. $\mathrm{HC}_{3} \mathrm{~N}$ and $\mathrm{C}_{6} \mathrm{H}_{6}$ were strongly depleted at altitudes lower than $250 \mathrm{~km}(0.1 \mathrm{mbar})$ likely due to their condensation. Because of their steep VMR meridional gradient near the south pole, these molecules can be regarded as the best tracers of the polar vortex boundary. In 2015, their VMR cross sections were compatible with a vortex boundary that was more confined toward the south pole between 300 and $400 \mathrm{~km}$ than at higher and lower altitudes. This gave it an anvil shape for the enriched zone on the maps of Figs. C.3, C.4, with a "tongue" of enriched air extending below $250 \mathrm{~km}$ toward lower latitudes. At 1 mbar (Figs. A.6-A.8) this tongue would correspond to a photochemical compound enriched torus enshrouding the polar region and its stratospheric polar cloud. We note that the tongue is also observed for $\mathrm{CH}_{3} \mathrm{C}_{2} \mathrm{H}$ (Figs. A.4 and C.2), $\mathrm{C}_{4} \mathrm{H}_{2}$ (A.3 and C.2), HCN (A.2 and C.1), and $\mathrm{C}_{2} \mathrm{H}_{2}$ (3 and C.1). In September 2015, using the boundary shape of the polar enriched zone we can deduce that the polar vortex was less confined around the south pole and that its boundary seemed to have extended down to almost $60^{\circ} \mathrm{S}$ around $300 \mathrm{~km}$. At this date, and in January 2016, the meridional VMR gradients of $\mathrm{C}_{2} \mathrm{H}_{4}, \mathrm{C}_{6} \mathrm{H}_{6}$, and $\mathrm{HC}_{3} \mathrm{~N}$ were less steep than in March 2015 and their enhancements extended down to $30^{\circ} \mathrm{S}$ at high altitude (higher than $300 \mathrm{~km}$ ). While later (in May 2017 ) the same high-altitude VMR values were observed beyond $60^{\circ} \mathrm{S}$. This suggests that the polar vortex was at its tightest latitudinal confinement in March 2015, then extended between March and September 2015, and retracted again in early 2016. This evolution is similar to the variation of the horizontal extension of the southern polar cloud observed by Cassini/VIMS in the same period (see Le Mouélic et al. 2018, their Fig. 4).

In the northern hemisphere, at latitudes higher than $60^{\circ} \mathrm{N}$ and in the $150-300 \mathrm{~km}$ altitude range, these molecules were enriched by factors of 10 to 100 compared to neighboring latitudes and 
altitudes. In March $2015 \mathrm{HC}_{3} \mathrm{~N}$ seemed to present a second enriched zone above $350 \mathrm{~km}$. The $\mathrm{C}_{2} \mathrm{H}_{4}$ VMR showed the most complex vertical profile at high-northern latitudes with several enriched zones, one in the $250-300 \mathrm{~km}$ range and another below $150 \mathrm{~km}$ observed in March 2015 and February 2016, while some enrichment was also observed in September 2015 at altitudes higher than $400 \mathrm{~km}$.

\subsubsection{Haze mass mixing ratio meridional cross sections}

We determined the haze mass mixing ratio from the retrieved haze optical depth assuming aerosol composed of 3000 monomers of $0.05 \mu \mathrm{m}$ radius each (Tomasko et al. 2008) with a bulk density of $0.6 \mathrm{~g} \mathrm{~cm}^{-3}$. Haze mass mixing ratio cross sections determined from the $600-620 \mathrm{~cm}^{-1}$ continuum thermal emission (FP3 spectra) are displayed in Figs. 4 and C.4 (middle). They are also compared in Fig. C.4 with the haze mass mixing ratio determined from the $1000-1100 \mathrm{~cm}^{-1}$ range acquired by the FP4. Uncertainties on the continuum at very low radiance levels are responsible for the differences in the meridional cross sections inferred from both focal planes. The haze spatial distribution displays similarities with those of the trace gases; it is strongly enriched in the southern polar vortex (by at least a factor of 10 at $400 \mathrm{~km}, 10^{-2}$ mbar) compared to mid-southern latitudes.

At high-northern latitudes the haze is also enriched in a confined region beyond $50^{\circ} \mathrm{N}$ and between 1 and 0.1 mbar (200$300 \mathrm{~km}$ ), like most of the molecules studied here. Like $\mathrm{C}_{2} \mathrm{H}_{2}$, $\mathrm{HCN}$, and $\mathrm{C}_{2} \mathrm{H}_{6}$, the haze mass mixing ratio is depleted in the northern mesosphere in November 2015, June 2016, and also probably in February 2017, while the mesosphere in May 2015 and September 2017 was enriched in haze. In September 2017, an enriched haze layer was inferred at $10^{-2}$ mbar $(400 \mathrm{~km})$ between $20^{\circ} \mathrm{N}$ and $60^{\circ} \mathrm{N}$. This last observation is compatible with the ISS observation of a haze layer at $\sim 400 \mathrm{~km}$ between $25^{\circ} \mathrm{N}$ and $50^{\circ} \mathrm{N}$ at the same date (Seignovert et al. 2020).

The haze mass mixing ratio profile globally increases with height because aerosols are produced in the upper atmosphere and are removed by sedimentation. Interestingly, in March and September 2015, we inferred a depletion in haze in the southern polar region below the 0.1 mbar level, where $\mathrm{C}_{6} \mathrm{H}_{6}, \mathrm{HC}_{3} \mathrm{~N}$, and $\mathrm{HCN}$ were condensing. This suggests that haze is efficiently removed in this region, probably because aerosols serve as condensation seeds for condensing molecules.

\subsection{Summary}

In summary, in the second part of the southern fall, all photochemical compounds were enriched at the south pole by the subsiding branch of the global circulation cell. By assuming that this enriched air was confined at high-southern latitudes by the polar vortex, the changes in the meridional cross sections of the VMR can be seen as tracers of the horizontal and vertical changing structure of the southern polar vortex. Photochemical species high enhancements combined with the unusually low polar temperatures observed in the deep stratosphere resulted in condensation between 0.1 and 0.03 mbar (240-280 km) of $\mathrm{HCN}, \mathrm{HC}_{3} \mathrm{~N}$, $\mathrm{C}_{6} \mathrm{H}_{6}$, and possibly $\mathrm{C}_{4} \mathrm{H}_{2}$ in March $2015\left(L_{s}=66^{\circ}\right)$. These molecules were observed to condense progressively deeper from the south pole. Right outside this condensation zone, the subsidence transported the enriched air toward the mid-latitude deep stratosphere probably forming a torus of enriched air enshrouding the polar vortex at $200 \mathrm{~km}$ and below.

At high-northern latitudes, stratospheric enrichments remaining from the previous winter were observed below $300 \mathrm{~km}$ between 2015 and May $2017\left(L_{s}=90^{\circ}\right)$ for all chemical compounds, and up to September $2017\left(L_{s}=93^{\circ}\right)$ for $\mathrm{C}_{2} \mathrm{H}_{2}, \mathrm{C}_{2} \mathrm{H}_{4}$, $\mathrm{CH}_{3} \mathrm{C}_{2} \mathrm{H}, \mathrm{C}_{3} \mathrm{H}_{8}$, and $\mathrm{C}_{4} \mathrm{H}_{2}$. These enrichments were observed at different altitudes depending on the molecule. It was observed at 0.1-0.5 mbar (250-300 km) for the haze, $\mathrm{C}_{2} \mathrm{H}_{2}, \mathrm{C}_{2} \mathrm{H}_{4}$ (with a more complex vertical structure for $\left.\mathrm{C}_{2} \mathrm{H}_{4}\right), \mathrm{CH}_{3} \mathrm{C}_{2} \mathrm{H}, \mathrm{C}_{4} \mathrm{H}_{2}$, and $\mathrm{HCN}$. This pressure region approximately corresponds to the location of the stratopause. In 2015 and 2016, the corresponding $\mathrm{C}_{3} \mathrm{H}_{8}$ enrichment zone was observed slightly deeper, in the $0.3-$ 1 mbar pressure range (150-250 km), and even deeper for $\mathrm{HC}_{3} \mathrm{~N}$, which was enriched in the 0.5-2 mbar range. $\mathrm{C}_{6} \mathrm{H}_{6}$ was also enriched, but only deeper than the 1 mbar level. In September 2017 these local enhancements were less pronounced than earlier for $\mathrm{C}_{2} \mathrm{H}_{2}, \mathrm{C}_{4} \mathrm{H}_{2}, \mathrm{CH}_{3} \mathrm{C}_{2} \mathrm{H}, \mathrm{HC}_{3} \mathrm{~N}$, and $\mathrm{HCN}$, and no longer observed for $\mathrm{C}_{2} \mathrm{H}_{6}$ and $\mathrm{C}_{6} \mathrm{H}_{6}$, which suggests a change in the northern polar dynamics near the summer solstice.

In the mesosphere, $\mathrm{C}_{2} \mathrm{H}_{2}, \mathrm{HCN}$, and $\mathrm{C}_{2} \mathrm{H}_{6}$ were depleted from the north pole to the mid-southern latitudes, while $\mathrm{C}_{4} \mathrm{H}_{2}$, $\mathrm{C}_{3} \mathrm{H}_{4}, \mathrm{C}_{2} \mathrm{H}_{4}$, and $\mathrm{HC}_{3} \mathrm{~N}$ seem to have been enriched in the same region. In the deep stratosphere, all molecules except $\mathrm{C}_{2} \mathrm{H}_{4}$ were depleted due to their condensation sink located deeper than 5 mbar outside the southern polar vortex. The HCN, $\mathrm{C}_{4} \mathrm{H}_{2}$, and $\mathrm{CH}_{3} \mathrm{C}_{2} \mathrm{H}$ VMR cross section contours showed steep slopes near mid-latitudes or close to the equator, which can be explained by upwelling air in this region. Upwelling is also supported by the $\mathrm{C}_{2} \mathrm{H}_{4}$ enriched air observed in the northern hemisphere.

\section{Discussion}

\subsection{Thermal field}

In the deep stratosphere, our results can be compared with those of Teanby et al. (2019), who inferred the temperature seasonal evolution from CIRS nadir spectra acquired at a spectral resolution of $3 \mathrm{~cm}^{-1}$. The temperatures derived in our study at 0.1 , 1 , and 5 mbar are overall very consistent with their results from 2015 to 2016. A comparison of our thermal field at 5 mbar with results from Sylvestre et al. (2020), who derived temperature at 6 mbar from far-infrared thermal emission acquired by CIRS (using focal plane 1), also shows good consistency.

In our previous study focusing on Titan's early spring from July 2009 to May 2013 (Vinatier et al. 2015), we were able to infer the pole-to-pole thermal field from December 2009 to February 2012. We recall that from 2012 to 2015 the Cassini orbit inclination prevented us from probing Titan's polar limbs. The derived thermal field in March 2015 differs greatly from that in 2012 mostly at polar latitudes. The strongest seasonal changes occurred at high-southern latitudes, where a $30 \mathrm{~K}$ decrease was derived between 2012 and 2015 in the deep stratosphere together with a $15 \mathrm{~K}$ increase at $2 \times 10^{-3}$ mbar. The seasonal evolution of the thermal field at high-southern latitudes results from a complex interplay between adiabatic heating from the descending branch of the circulation cell and enhanced radiative cooling from the enriched molecules, as detailed by Teanby et al. (2017). In 2015, the southern polar vortex was already well established.

The high-northern latitude stratopause was observed about one pressure decade deeper in 2015 than in 2012 and was $5 \mathrm{~K}$ warmer. The deeper location of the stratopause at high-northern latitudes could be explained by the combined effect of adiabatic cooling at the $10^{-2}-10^{-3}$ mbar pressure range by the upwelling branch of the global circulation, and the increasing mid-spring insolation. 
The mid- and low-latitude stratopause was located at the same pressure in 2012 (Vinatier et al. 2015) and 2015, but the $10-20^{\circ} \mathrm{N}$ stratopause was $2 \mathrm{~K}$ colder in 2015 than in 2012 at 0.2-0.3 mbar, which may result from Saturn's orbit eccentricity (Bézard et al. 2018).

The temperature profiles we derived can be compared with those of Mathé et al. (2020) inferred from CIRS limb spectra acquired at $0.5-\mathrm{cm}^{-1}$ spectral resolution at comparable latitudes and time. Their dataset probed the same pressure region as ours. The agreement with their mid-northern and highnorthern latitude profiles in 2015 and 2016 is very good. Our derived temperatures are also consistent below the mesosphere with their equatorial and mid-southern latitude profiles. However, we derived mesospheric temperatures that were higher by 5-10 K than their 2017 equatorial values and their 2016-2017 mid-southern values. At the south pole, our thermal profiles are also in very good agreement with theirs at similar latitudes and longitudes. For instance, in March 2015 Fig. C.1 shows that the thermal field is not symmetrical around the south pole with the thermal profile at $81^{\circ} \mathrm{S} 255^{\circ} \mathrm{W}(17: 58$ local time, right-hand side of the pole in Fig. C.1) showing a stratopause (at $400 \mathrm{~km}$ ) $12 \mathrm{~K}$ warmer than at $78^{\circ} \mathrm{S} 119^{\circ} \mathrm{W}(02: 52$ local time, left-hand side of the pole in Fig. C.1). As this later thermal profile is in agreement with that inferred by Vinatier et al. (2018) and with the results of Mathé et al. (2020) in the $10^{-2}-10^{-4}$ mbar range, which were extracted from other CIRS limb data acquired at the same date, longitude, and $15 \mathrm{~h}$ apart, it strengthens our derived asymmetry of the thermal field around the south pole in March 2015. This asymmetry probably results from the different insolation conditions between these two limb observations.

\subsection{Molecular gas mixing ratio meridional cross sections}

Our VMR profiles can be compared with the retrieved profiles of Mathé et al. (2020), who used CIRS limb spectra acquired at $0.5 \mathrm{~cm}^{-1}$ over the entire Cassini mission. Our retrieved VMR profiles are overall in very good agreement with their results. For instance, at high-northern latitude, they also inferred similar local VMR maxima for $\mathrm{C}_{2} \mathrm{H}_{2}$ and $\mathrm{HCN}$ in the $0.1-$ 1 mbar pressure range, they derived a local VMR minimum at $\sim 0.005$ mbar with equivalent VMR values. Other VMR profiles that we derived in this study are also consistent with their results, except at $81^{\circ} \mathrm{S}-125^{\circ} \mathrm{W}$ in March 2015 as they retrieved $\mathrm{C}_{2} \mathrm{H}_{2}$ and HCN VMR 10 times higher than ours at and above $10^{-4}$ mbar, while our profiles agree deeper than this pressure level for $\mathrm{C}_{2} \mathrm{H}_{2}$. However, at this location, other molecular VMRs are consistent within the error bars. These observations have larger uncertainties than any other because of the low temperature there, which can explain discrepancies between the two studies.

The mixing ratios we retrieved around 1 mbar can be compared with those retrieved by Teanby et al. (2019) and Coustenis et al. (2020), who inferred VMRs from CIRS nadir spectra acquired at 2.5 and $0.5 \mathrm{~cm}^{-1}$ spectral resolution, respectively. Common molecules between our study and Teanby et al. (2019) are $\mathrm{C}_{2} \mathrm{H}_{2}, \mathrm{C}_{2} \mathrm{H}_{4}, \mathrm{C}_{2} \mathrm{H}_{6}, \mathrm{CH}_{3} \mathrm{C}_{2} \mathrm{H}, \mathrm{C}_{4} \mathrm{H}_{2}, \mathrm{HCN}$, and $\mathrm{HC}_{3} \mathrm{~N}$; Coustenis et al. (2020) additionally retrieved VMR of $\mathrm{C}_{3} \mathrm{H}_{8}$ and $\mathrm{C}_{6} \mathrm{H}_{6}$. As vertical information is very limited from nadir observations, both studies assumed constant-with-height mixing ratio profiles above the condensation level, which makes a detailed comparison partially meaningful as our derived profiles show strong vertical variations with altitude. Nevertheless, around 1 mbar the relative decrease in molecular VMRs at the north pole and their relative increase at the south pole (Teanby et al. 2019) in the 2015-2017 period in is overall agreement with our results. However, because of their constant-with-height VMR assumption, these authors could not derive the local enhancement that we inferred at high-northern latitude in the 1-0.1 mbar pressure range. In general, our stratospheric (in the 5-0.5 mbar range) inferred VMR are in good agreement with those derived by Coustenis et al. (2020).

We derived the highest VMR in the south pole mesosphere in March 2015, when the polar vortex was at its tightest latitudinal confinement. Our derived VMRs of $\mathrm{C}_{2} \mathrm{H}_{2}, \mathrm{HCN}, \mathrm{CH}_{3} \mathrm{C}_{2} \mathrm{H}$, and $\mathrm{C}_{4} \mathrm{H}_{2}$ inside the polar vortex at $600 \mathrm{~km}\left(2 \times 10^{-5}\right.$ mbar $)$ are similar to the VMRs measured in situ by the Cassini Ion Neutral Mass Spectrometer (INMS) above $1000 \mathrm{~km}$ (Vuitton et al. 2007; Cui et al. 2009; Magee et al. 2009). This observation, also reported by Mathé et al. (2020), suggests that subsidence at the south pole transports enriched air from altitudes much higher than $600 \mathrm{~km}$. Additionally, the derived thermal zonal wind (see Sect. 5.5) also supports a more confined polar vortex in March 2015 with winds of $\sim 240 \mathrm{~m} \mathrm{~s}^{-1}$ reaching at least $600 \mathrm{~km}$, with subsidence therefore necessarily coming from higher altitudes.

The only molecule among all those studied here that does not condense in Titan's atmosphere is $\mathrm{C}_{2} \mathrm{H}_{4}$. This explains why it is enriched in the deep stratosphere by advection from the fall-winter polar region towards the spring-summer pole in the deep atmosphere by the deep branch of the global circulation (Vatant d'Ollone 2020). Additionally, $\mathrm{C}_{2} \mathrm{H}_{4}$ is photodissociated below $500 \mathrm{~km}$ and reacts with $\mathrm{H}$ atoms to form $\mathrm{C}_{2} \mathrm{H}_{5}$ (Vuitton et al. 2019), which can explain its depletion at mid- and low latitudes above $150 \mathrm{~km}$. The global northern hemisphere enrichment compared to the southern hemisphere below $300 \mathrm{~km}$ observed since November $2015\left(L_{s}=73^{\circ}\right)$ could be explained by a global vertical transport of this molecule at mid- and high-northern latitudes, which is compatible with the spring and summer ascending branch predicted by GCMs (e.g., Vatant d'Ollone 2020). The complex northern polar structure of its VMR meridional cross section suggests a complex circulation pattern, perhaps including two small circulation cells that could have coexisted in 2015 and 2016, keeping the $\mathrm{C}_{2} \mathrm{H}_{4}$ enrichments in two distinct altitude ranges (one around 0.1 mbar and another one deeper than 1 mbar).

\subsection{Local mesospheric minimum VMR of $\mathrm{C}_{2} \mathrm{H}_{2}, \mathrm{C}_{2} \mathrm{H}_{6}$, and $\mathrm{HCN}$}

According to Vuitton et al. (2019), the predicted lifetimes of $\mathrm{C}_{2} \mathrm{H}_{2}, \mathrm{C}_{2} \mathrm{H}_{6}$, and $\mathrm{HCN}$ in the mesosphere are mostly controlled by vertical transport timescales, resulting in lifetimes of 14 Titan days (230 days) at $500 \mathrm{~km}\left(\sim 10^{-3}\right.$ mbar $)$ for $\mathrm{C}_{2} \mathrm{H}_{2}, 100$ Titan days for $\mathrm{C}_{2} \mathrm{H}_{6}$, and 1000 Titan days for $\mathrm{HCN}$. Chemical lifetimes decrease with altitude, and it is possible that the observed mesospheric depleted air comes from higher levels where these molecules are more efficiently photodissociated. Assuming that the $\mathrm{C}_{2} \mathrm{H}_{2}$ - and $\mathrm{HCN}$-depleted air observed at $80^{\circ} \mathrm{N}$ on May 9 , 2015 , was horizontally advected to $40^{\circ} \mathrm{S}$ on September 29, 2015, would imply an horizontal advection of $50 \mathrm{~cm} \mathrm{~s}^{-1}$, a value higher but not so far from the $0.1-0.2 \mathrm{~m} \mathrm{~s}^{-1}$ predicted by the IPSL Titan GCM in diurnal average (Vatant d'Ollone 2020; Vatant d'Ollone et al. 2018). Using the continuity equation, this horizontal velocity would imply a subsidence vertical velocity of $\sim 3 \mathrm{~mm} \mathrm{~s}^{-1}$ inside the southern polar vortex at $10^{-2}$ mbar $(\sim 400 \mathrm{~km})$, which is consistent with estimations of Teanby et al. (2017) in 2015 using a radiative model and from their derived mixing ratio profiles. Another possibility to explain the presence of this depleted zone could be diurnal effects, which is at odds with the conclusion of Vuitton et al. (2019). We observe a correlation between 
the mesospheric temperature maximum observed during the day (displayed in yellow and orange in Figs. 2 and C.1) and the lower VMRs of $\mathrm{C}_{2} \mathrm{H}_{2}, \mathrm{C}_{2} \mathrm{H}_{6}$, and $\mathrm{HCN}$. Instead, during the nighttime the mesospheric VMRs seemed to be enhanced. This hypothesis needs other data analysis to be confirmed or refuted.

\subsection{Haze extinction meridional cross sections: comparison with ISS observations}

Seignovert et al. (2020) derived the seasonal variation of Titan's haze extinction from October 2004 to September 2017 in the $350-550 \mathrm{~km}$ altitude range from the analysis of the Cassini Image Sub-System Narrow Angle Camera in the CL1-UV3 UV filter at $338 \mathrm{~nm}$. We can compare the spatial distribution of extinction we derived from CIRS in the $350-450 \mathrm{~km}$ range, which is generally the overlap altitude range probed by UV and thermal infrared observations. Seignovert et al. (2020) inferred complex temporal variation of the haze extinction in the stratopause and mesospheric levels with high temporal variability. In order to compare haze extinction coefficients derived from CIRS observations at $1090 \mathrm{~cm}^{-1}$ and the UVIS ones at $338 \mathrm{~nm}\left(29586 \mathrm{~cm}^{-1}\right)$, we applied a multiplicative factor equal to $1.22 \times 10^{-3}$ derived from the far-IR to UV spectral dependence of the aerosol cross sections of Vinatier et al. (2012, see Fig. 2 therein). A comparison of our haze extinction coefficient maps with those derived from ISS observations acquired at similar dates is displayed in Appendix D. In the overlapping altitude range, agreement is overall within a factor of 2 , which is typically the uncertainty on the haze extinction coefficient derived from CIRS limb spectra above $350 \mathrm{~km}$. The largest difference is inferred in September 2017, which corresponds to an ISS observation with a limited latitudinal coverage for which Titan's center adjustment, and therefore the altitude grid extraction, is not as well constrained as for other ISS datasets (see Seignovert et al. 2020).

\subsection{Thermal winds}

Performing a scale analysis for Titan's parameters of the different terms of the momentum equation in spherical coordinates projected over the northward direction gives the remaining terms:

$2 \Omega \sin (\phi) u+\frac{\tan (\phi)}{r} u^{2}+\left.\frac{1}{\rho r} \frac{\partial p}{\partial \phi}\right|_{z}=0$.

Here $u$ is the zonal wind velocity (eastward), $\Omega$ the rotation rate of Titan, $r$ the sum of Titan's radius and altitude, $\phi$ the latitude, $p$ the pressure, and $\rho$ the atmospheric number density. Frictional forces can be neglected in the stratosphere. Appendix E gives solutions of this equation that we applied to calculate $u$ from the meridional pressure gradient. Titan's zonal winds were previously determined from the thermal field derived from CIRS limb and nadir observations by Flasar et al. (2005) and Achterberg et al. (2008) during Titan's northern winter, by Achterberg et al. (2011) from winter to northern spring, and extended by Achterberg et al. (2018) over early northern summer but deeper than 0.05 mbar as they used CIRS nadir datasets. Our zonal wind integration differs from these previous studies as we did not integrate the thermal wind equation over cylinders concentric around Titan's rotation axis (Flasar et al. 2005), but instead performed integration in spherical coordinates (see Appendix E). We set the zonal wind at 5 mbar to $120 \mathrm{~m} \mathrm{~s}^{-1}$, which is compatible with in situ measurements acquired by the Doppler Wind
Experiment aboard Huygens during the probe descent (Bird et al. 2005; Folkner et al. 2006).

Figure 5 shows the meridional cross sections of zonal wind velocity (eastward) derived from the thermal fields displayed in Fig. 2. During the studied period all the derived meridional cross sections of zonal wind show a clear asymmetry between the northern and southern hemispheres with zonal winds much faster in the southern hemisphere than in the northern one. If we consider that the southern polar vortex boundary is characterized by the strongest zonal winds around the south pole (circumpolar jet reaching 240-280 $\mathrm{m} \mathrm{s}^{-1}$ ), our derived zonal wind maps in March 2015 and September 2015 show that the polar vortex was confined to latitudes higher than $70^{\circ} \mathrm{S}$ in March and $60^{\circ} \mathrm{S}$ in September. This horizontal extension is consistent with results from the VMR cross sections that display the same latitudinal extension of the enriched zone between the two dates (see, e.g., Fig. A.2). In 2016 the high-southern latitude jet seemed to slow down while migrating toward low-southern latitudes in June 2016 and February 2017. A less pronounced zonal wind local maximum was derived at $65^{\circ} \mathrm{S}$ at $\sim 10^{-2}$ mbar in June 2016 and February and May 2017. Such temporal evolution of zonal winds can explain the temporal change in the VMR enrichment patterns at high-southern latitude described in Sect. 4.2, confirming that they are linked to the polar vortex changing structure.

In all of our derived stratospheric wind maps, the northern zonal winds are much weaker than the southern hemisphere winds with values typically lower than $160 \mathrm{~m} \mathrm{~s}^{-1}$, which is (given the studied period) totally consistent with momentum conservation in a superrotating atmosphere overturning cell leading to faster winds during autumn and winter in altitude. The northern wind speeds seemed to be globally higher in the deep stratosphere (deeper than $10^{-1}$ mbar) than above the stratopause (above $10^{-1}$ mbar). This last point, added to the fact mentioned previously that the stratopause seems to be an area of limited mixing for some species, supports the idea (supported by models such as the ISPL Titan GCM) that the stratopause could act as a separation between stratospheric and mesospheric circulation pattern (Vatant d'Ollone 2020; Vatant d'Ollone et al. 2018).

We can compare our derived zonal wind cross section with the one derived by Achterberg et al. (2018) in 2016 and 2017. Their maps are determined below the 0.05 mbar level because they were calculated from thermal fields inferred from CIRS nadir observations. Below this pressure range, our derived zonal wind horizontal and vertical structures are in good agreement.

Lellouch et al. (2019) determined zonal wind velocities in July and August 2016 from the Doppler shifts of submillimeter lines for several molecules acquired by ALMA (Atacama Large Submillimeter Array) interferometer. From all their studied molecules, which probe different levels in the atmosphere, they derived wind maps with faster zonal winds in the southern hemisphere than in the northern hemisphere, along with an equatorial jet. Our derived thermal wind structures in May 2016 are overall in very good agreement with their measurements as we derive wind values at $20^{\circ} \mathrm{S}\left(240-260 \mathrm{~m} \mathrm{~s}^{-1}\right.$ in the $300-600 \mathrm{~km}$ range, or in the $0.1-10^{-4}$ mbar pressure range) comparable to their fitted values assuming solid-body rotation $\left(210 \mathrm{~m} \mathrm{~s}^{-1}\right.$ between 200 and $400 \mathrm{~km}$, and $260-290 \mathrm{~m} \mathrm{~s}^{-1}$ between 400 and $700 \mathrm{~km}$ ). Our larger zonal wind observed at low-southern latitudes in May 2016 (and probably also in February and May 2017) is consistent with the Lellouch et al. (2019) direct detection of an equatorial jet in August 2016. It should be noted, however, that for latitudes lower than $20^{\circ}$ the $\sqrt{\frac{1}{\tan (\phi)}}$ 
S. Vinatier et al.: Seasonal variations in Titan's atmosphere close to the northern summer solstice
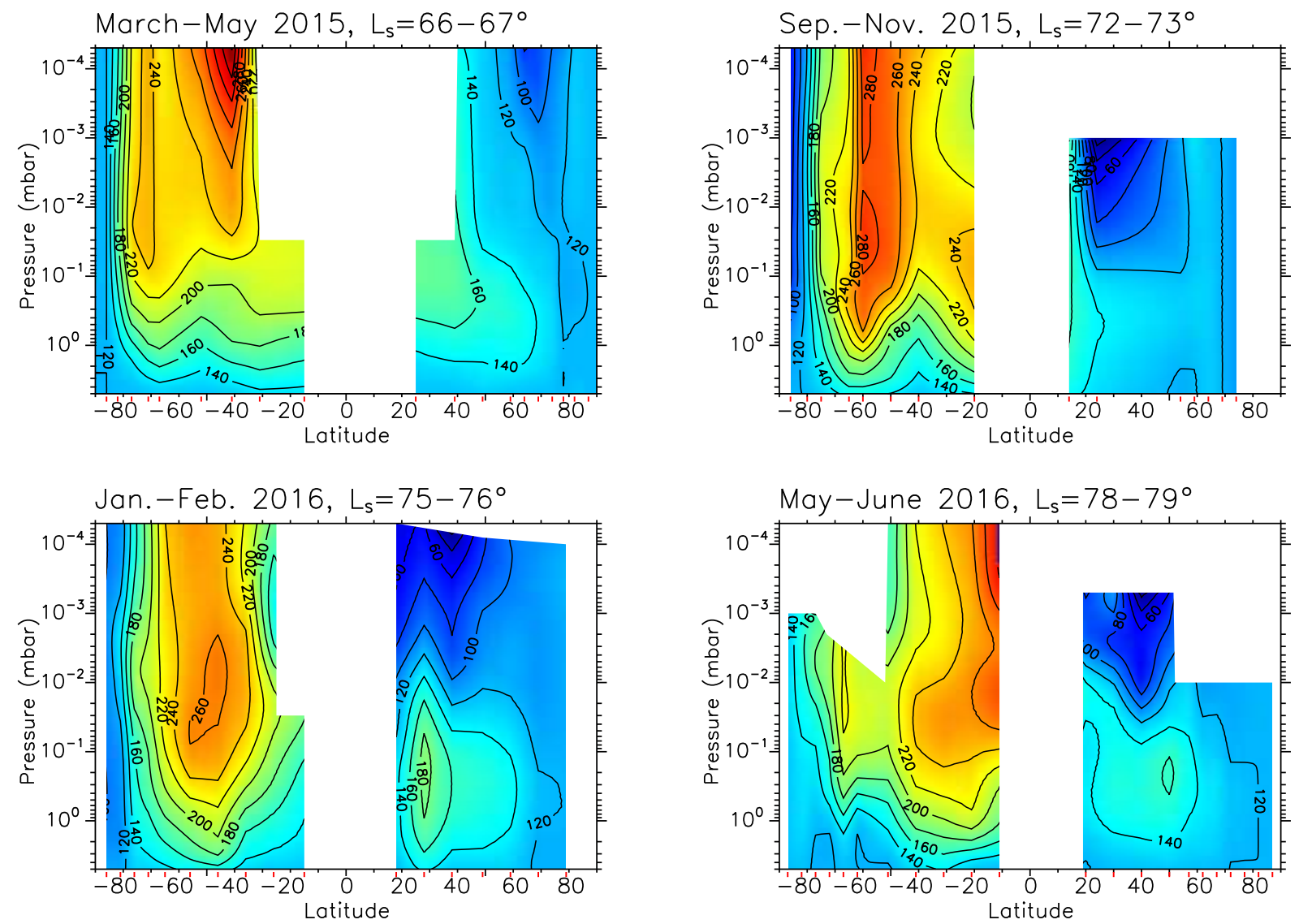

Feb. $2017, L_{s}=87^{\circ}$
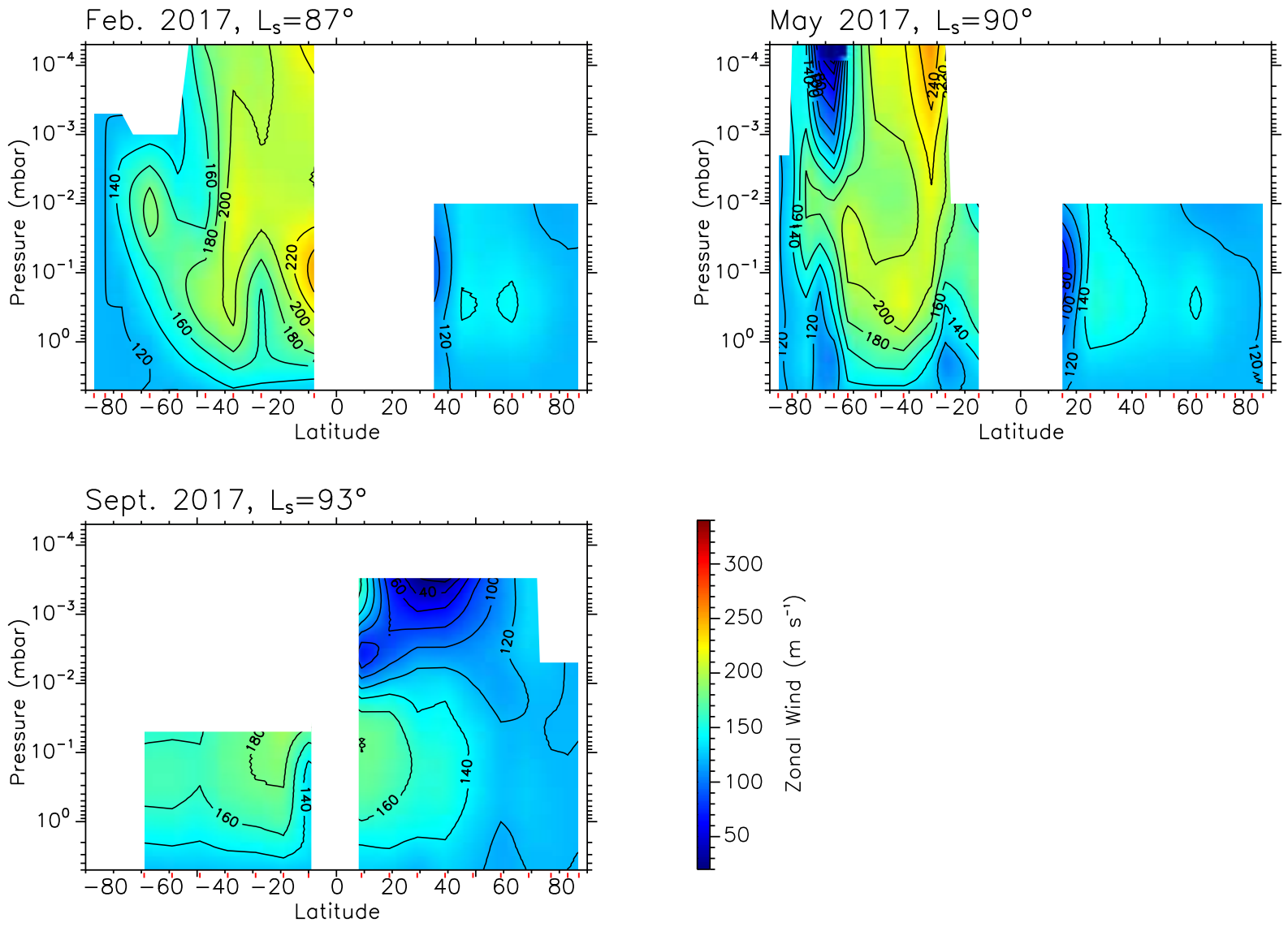

Fig. 5. Zonal wind cross sections from March 2015 to September 2017. Contours are given every $20 \mathrm{~m} \mathrm{~s}^{-1}$; the color scale is the same for all plots. Regions without information are represented in white. They correspond to missing data, pressure levels not probed by nadir observations, or regions not used to perform the wind calculation at low latitudes. 

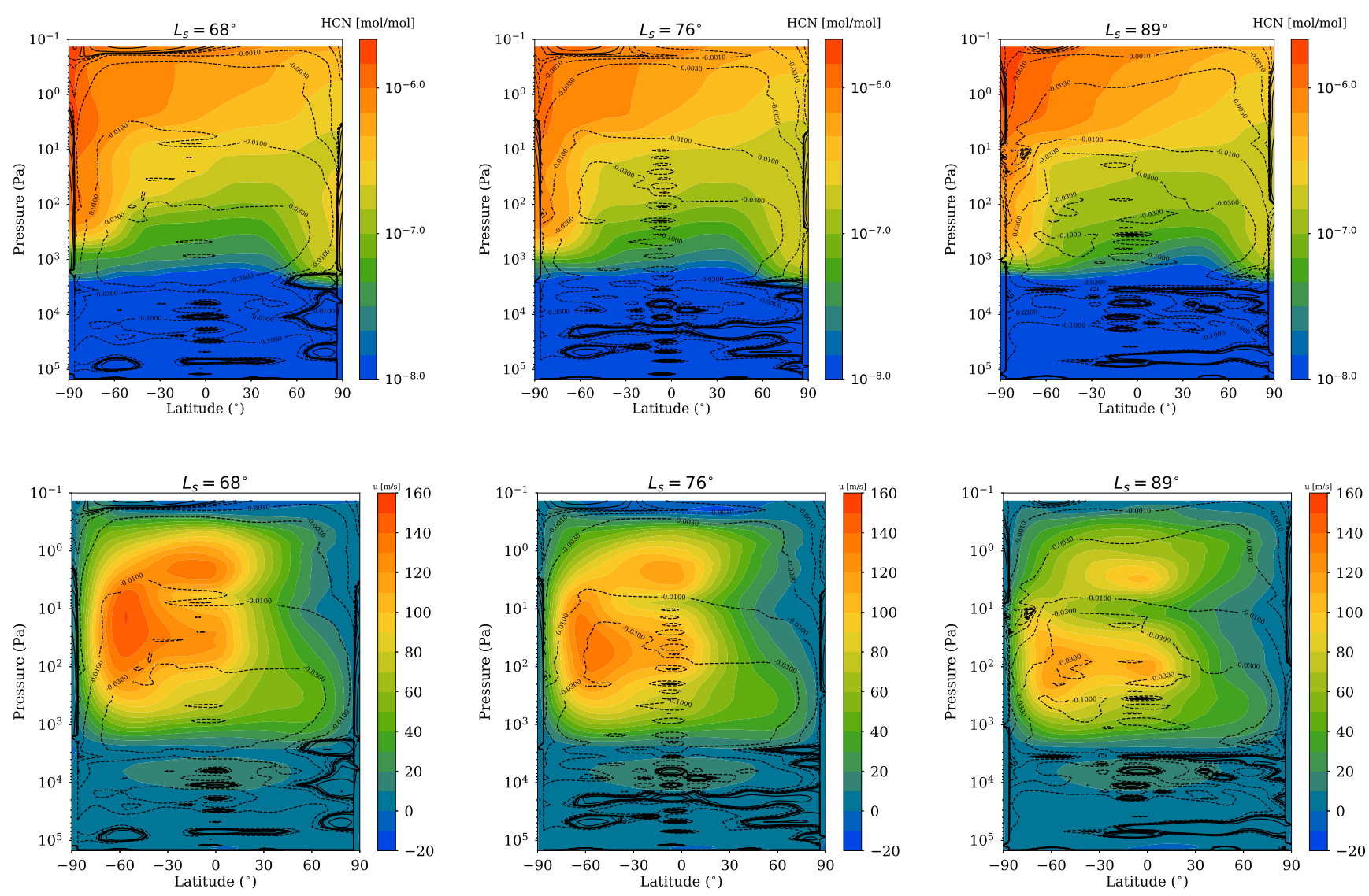

Fig. 6. Predictions of the IPSL Titan GCM for HCN volume mixing ratio (top) and the zonal wind (m $\mathrm{s}^{-1}$, bottom) for $L_{s}=68,76$, and $89^{\circ}$. Meridional circulation is superimposed with stream functions $\left(10^{9} \mathrm{~kg} \mathrm{~s}^{-1}\right)$ in solid lines for clockwise circulation and dotted lines for counterclockwise.

term in the zonal wind solution (see Appendix E) can amplify uncertainties on our derived wind profiles.

Our results show that two years before the southern winter solstice, the southern polar vortex structure was changing within a few Titan days, resulting in more or less pronounced molecular enhancements inside the polar vortex. One year before the summer solstice, two jets are derived in the southern stratosphere near $70^{\circ} \mathrm{S}$ and at mid-southern latitudes.

\subsection{Comparison with the Institut Pierre Simon Laplace Titan Global Climate Model}

Our VMR meridional cross sections can be compared with the predictions of the IPSL Titan GCM. This GCM, which has been recently upgraded to take into account coupling between chemistry, radiative transfer, haze microphysics, and global dynamics (Vatant d'Ollone 2020; Vatant d'Ollone et al. 2018), is currently the most complete. It models processes occurring in the middle atmosphere of Titan.

Figure 6 shows the predicted HCN VMR (top) and zonal wind (bottom) for $L_{s}=68^{\circ}, 76^{\circ}$, and $89^{\circ}$, with corresponding stream functions for each season. At mid- and low latitudes, the molecular depletion that we observe in the deep stratosphere is predicted by the GCM to result from air ascending from condensation levels (see the predicted VMR in the $10^{2}-10^{3} \mathrm{~Pa}$ region).

The northern winter residual circulation cell is predicted to extend from the north pole to $\sim 50^{\circ} \mathrm{N}$ in the deep stratosphere and decrease in size with time, before vanishing around the northern summer solstice. Seasonal change of this residual circulation cell was responsible for the maintenance of the local northern polar stratospheric enhancements during spring. This result from the GCM, although predicted at deeper levels than observed here, could explain our local gas VMR and haze mass mixing ratio enrichment at the north pole deeper than 0.1 mbar, which slowly dissipated after the northern summer solstice. The IPSL Titan GCM also predicts that the southern mesosphere is more enriched in photochemical products than the northern mesosphere. This tendency is also observed here; however, we inferred southern enhancements that are more confined around the south pole than the GCM prediction before the southern summer solstice. This difference can be explained by the GCM top layer located at $10^{-1} \mathrm{~Pa}$, which limits the vertical extension of the meridional circulation along with the horizontal resolution used in the model, which is too low to reproduce steep meridional gradients. Despite these limitations, the IPSL Titan GCM predicts a high-southern latitude mesosphere (above the $10 \mathrm{~Pa}$ level) that is more enriched than earlier in photochemical compounds near the summer solstice, combined with a light depletion of polar air deeper than $10 \mathrm{~Pa}$. This prediction is also in agreement with our observations, even though observed in a lower pressure range.

As in most of the models, predicted zonal winds for northern spring and summer are faster in the southern hemisphere than is the northern. Structure of the southern polar vortex is predicted to change within the observing time studied here, with a global decrease in zonal wind speed with time and development of a double equatorial jet, a stratospheric and a mesospheric. This predicted complex seasonal variation of the zonal wind is in very 
good agreement with our thermal zonal wind calculation, albeit with a pressure decade difference, which is again likely due to the vertical extension of the IPSL Titan GCM. Our derived equatorial thermal winds are much faster than those predicted by this GCM, which can be explained by their predicted thermal contrasts that are less pronounced than those inferred from CIRS data. Further discussions can be found in Vatant d'Ollone et al. (2018).

Acknowledgements. This work was funded by the French Centre National d'Etudes Spatiales (CNES) and the Programme National de Planétologie (INSU). M.S. and N.A.T. acknowledge support from the UK Science and Technology Facilities Council (grant number ST/MOO7715/1).

\section{References}

Achterberg, R. K., Conrath, B. J., Gierasch, P. J., Flasar, F. M., \& Nixon, C. A. 2008, Icarus, 194, 263

Achterberg, R. K., Gierasch, P. J., Conrath, B. J., Michael Flasar, F., \& Nixon, C. A. 2011, Icarus, 211, 686

Achterberg, R. K., Gierasch, P. J., Nixon, C. A., \& Flasar, F. M. 2018, in Cassini Science Symposium, 13th-17th August, Boulder, USA

Anderson, C. M., Samuelson, R. E., \& Nna-Mvondo, D. 2018, Space Sci. Rev., 214,125

Bézard, B., Vinatier, S., \& Achterberg, R. K. 2018, Icarus, 302, 437

Bird, M. K., Allison, M., Asmar, S. W., et al. 2005, Nature, 438, 800

Coustenis, A., Achterberg, R. K., Conrath, B. J., et al. 2007, Icarus, 189, 35

Coustenis, A., Jennings, D. E., Nixon, C. A., et al. 2010, Icarus, 207, 461

Coustenis, A., Jennings, D. E., Achterberg, R. K., et al. 2020, Icarus, 344, 113413

Cui, J., Yelle, R. V., Vuitton, V., et al. 2009, Icarus, 200, 581

de Kok, R. J., Teanby, N. A., Maltagliati, L., Irwin, P. G. J., \& Vinatier, S. 2014, Nature, 514, 65

Feofilov, A., Rezac, L., Kutepov, A., et al. 2016, Titan Aeronomy and Climate, 2

Flasar, F. M., Kunde, V. G., Abbas, M. M., et al. 2004, Space Sci. Rev., 115, 169

Flasar, F. M., Achterberg, R. K., Conrath, B. J., et al. 2005, Science, 308, 975

Folkner, W. M., Asmar, S. W., Border, J. S., et al. 2006, J. Geophys. Res. Planets, 111, E07S02

Jennings, D. E., Anderson, C. M., Samuelson, R. E., et al. 2012, ApJ, 761, L15

Jennings, D. E., Achterberg, R. K., Cottini, V., et al. 2015, ApJ, 804, L34

Jennings, D. E., Flasar, F. M., Kunde, V. G., et al. 2017, Appl. Opt., 56, 5274

Kunde, V. G., Ade, P. A., Barney, R. D., et al. 1996, Proc. SPIE, 2803, 162

Lebonnois, S., Burgalat, J., Rannou, P., \& Charnay, B. 2012, Icarus, 218, 707

Lebonnois, S., Flasar, F. M., Tokano, T., \& Newman, C. E. 2014, The general circulation of Titan's lower and middle atmosphere (Cambridge: Cambridge University Press), 122

Lellouch, E., Gurwell, M. A., Moreno, R., et al. 2019, Nat. Astron., 3, 614

Le Mouélic, S., Rodriguez, S., Robidel, R., et al. 2018, Icarus, 311, 371

Lora, J. M., Lunine, J. I., \& Russell, J. L. 2015, Icarus, 250, 516

Magee, B. A., Waite, J. H., Mandt, K. E., et al. 2009, Planet. Space Sci., 57, 1895

Mathé, C., Vinatier, S., Bézard, B., et al. 2020, Icarus, 344, 113547

Newman, C. E., Lee, C., Lian, Y., Richardson, M. I., \& Toigo, A. D. 2011, Icarus, 213,636
Nixon, C. A., Ansty, T. M., Lombardo, N. A., et al. 2019, ApJS, 244, 14 Seignovert, B., Rannou, P., West, R. A., \& Vinatier, S. 2020, ApJ, submitted Sung, K., Toon, G. C., Mantz, A. W., \& Smith, M. A. H. 2013, Icarus, 226, 1499 Sylvestre, M., Teanby, N. A., Vinatier, S., Lebonnois, S., \& Irwin, P. G. J. 2018 , A\&A, 609, A64

Sylvestre, M., Teanby, N. A., Vattant d'Ollone, J., et al. 2020, Icarus, 344, 113188 Teanby, N. A., Irwin, P. G. J., de Kok, R., et al. 2007, Icarus, 186, 364

Teanby, N. A., de Kok, R., Irwin, P. G. J., et al. 2008, J. Geophys. Res., 113 , E12003

Teanby, N. A., Irwin, P. G. J., Nixon, C. A., et al. 2012, Nature, 491, 732

Teanby, N. A., Bézard, B., Vinatier, S., et al. 2017, Nat. Comm., 8, 1586

Teanby, N. A., Sylvestre, M., Sharkey, J., et al. 2019, Geophys. Res. Letter, 46, 3079

Tomasko, M. G., Doose, L., Engel, S., et al. 2008, Planet. Space Sci., 56, 669

Vatant d'Ollone, J. 2020, PhD thesis, Sorbonne Université, Paris, France

Vatant d'Ollone, J., Lebonnois, S., \& Burgalat, J. 2018, European Planetary Science Congress (Berlin, Germany: TU Berlin), EPSC2018

Vinatier, S., Bézard, B., Fouchet, T., et al. 2007, Icarus, 188, 120

Vinatier, S., Bézard, B., Nixon, C. A., et al. 2010a, Icarus, 205, 559

Vinatier, S., Bézard, B., de Kok, R., et al. 2010b, Icarus, 210, 852

Vinatier, S., Rannou, P., Anderson, C. M., et al. 2012, Icarus, 219, 5

Vinatier, S., Bézard, B., Lebonnois, S., et al. 2015, Icarus, 250, 95

Vinatier, S., Schmitt, B., Bézard, B., et al. 2018, Icarus, 310, 89

Vuitton, V., Yelle, R. V., \& McEwan, M. J. 2007, Icarus, 191, 722

Vuitton, V., Yelle, R. V., Klippenstein, S. J., Hörst, S. M., \& Lavvas, P. 2019, Icarus, 324, 120

West, R. A., Del Genio, A. D., Barbara, J. M., et al. 2016, Icarus, 270, 399

${ }^{1}$ LESIA, Observatoire de Paris, Université PSL, CNRS, Sorbonne Université, Université de Paris, 5 place Jules Janssen, 92195 Meudon, France e-mail: sandrine.vinatier@obspm.fr

2 LATMOS IPSL, Sorbonne Université, UVSQ Paris-Saclay, CNRS, Paris, France

${ }^{3}$ Laboratoire de Météorologie Dynamique (LMD/IPSL), Sorbonne Université, ENS, PSL Research University, Ecole Polytechnique, IP Paris, CNRS, 4 Place Jussieu, 75252 Paris Cedex 05, France

${ }^{4}$ School of Physics and Astronomy, University of Leicester, University Road, Leicester, LE1 7RH, UK

5 Institut Villebon - Georges Charpak, Bat. 490, rue Hector Berlioz, Université Paris-Saclay, 91400 Orsay, France

${ }^{6}$ NASA/Goddard Space Flight Center, Code 693, Greenbelt, MD 20771, USA

7 Jet Propulsion Laboratory, California Institute of Technology, Pasadena, CA 91109, USA

${ }^{8}$ School of Earth Sciences, University of Bristol, Wills Memorial Building, Queen's Road, Bristol BS8 1 RJ, UK

${ }^{9}$ Department of Astronomy, University of Maryland, College Park, MD 20742, USA

10 ADNET Systems, Inc., Bethesda, Maryland 20817, USA 


\section{Appendix A: Additional figures}

Meridional cross sections of the volume mixing ratios of $\mathrm{C}_{2} \mathrm{H}_{6}$, $\mathrm{HCN}, \mathrm{C}_{4} \mathrm{H}_{2}, \mathrm{CH}_{3} \mathrm{CCH}, \mathrm{C}_{3} \mathrm{H}_{8}, \mathrm{HC}_{3} \mathrm{~N}, \mathrm{C}_{6} \mathrm{H}_{6}, \mathrm{C}_{2} \mathrm{H}_{4}$ and the haze extinction are displayed in Figs. A.1-A.9, respectively. 
S. Vinatier et al.: Seasonal variations in Titan's atmosphere close to the northern summer solstice
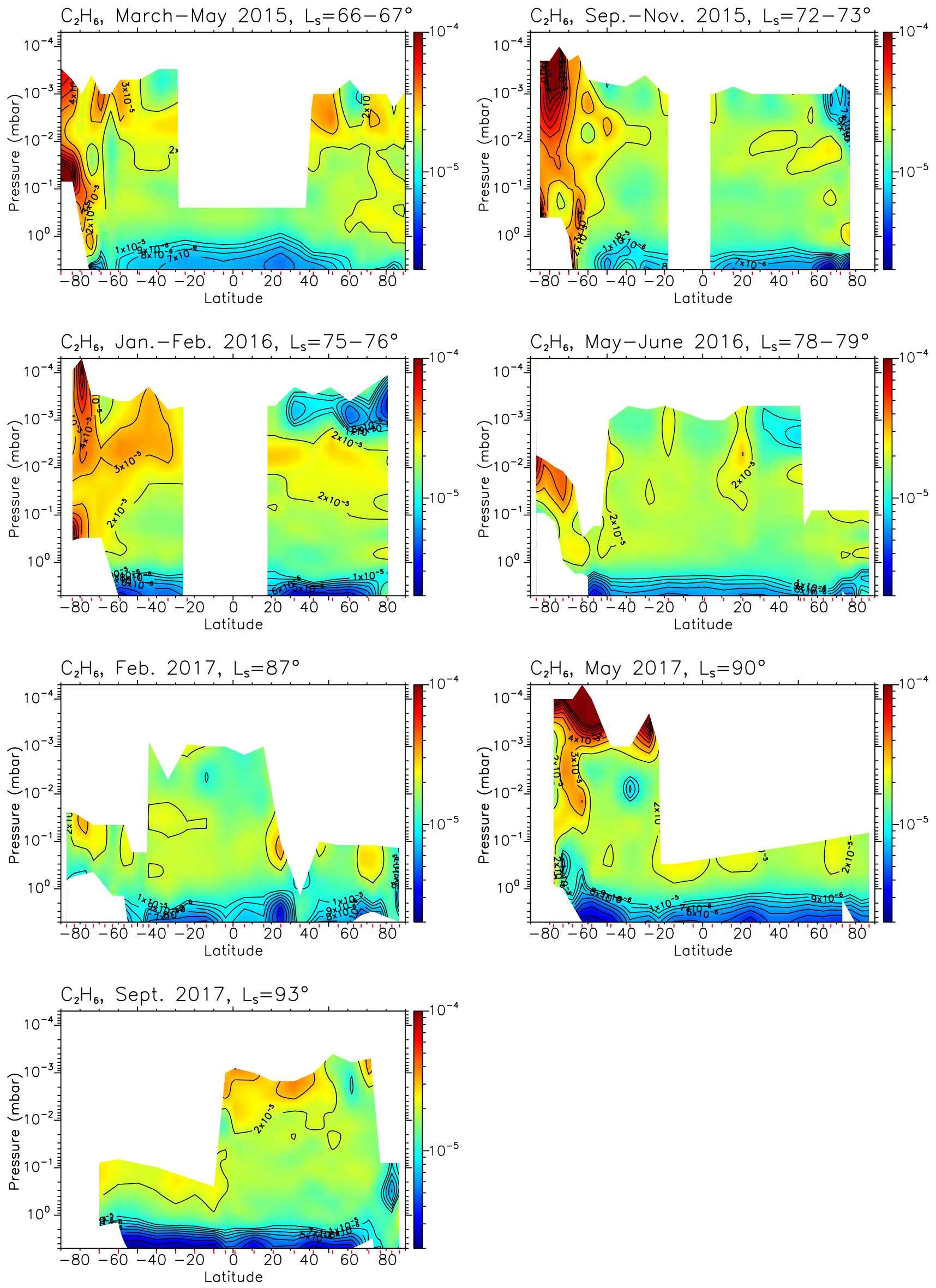

Fig. A.1. Same as Fig. 3, but for $\mathrm{C}_{2} \mathrm{H}_{6}$. 

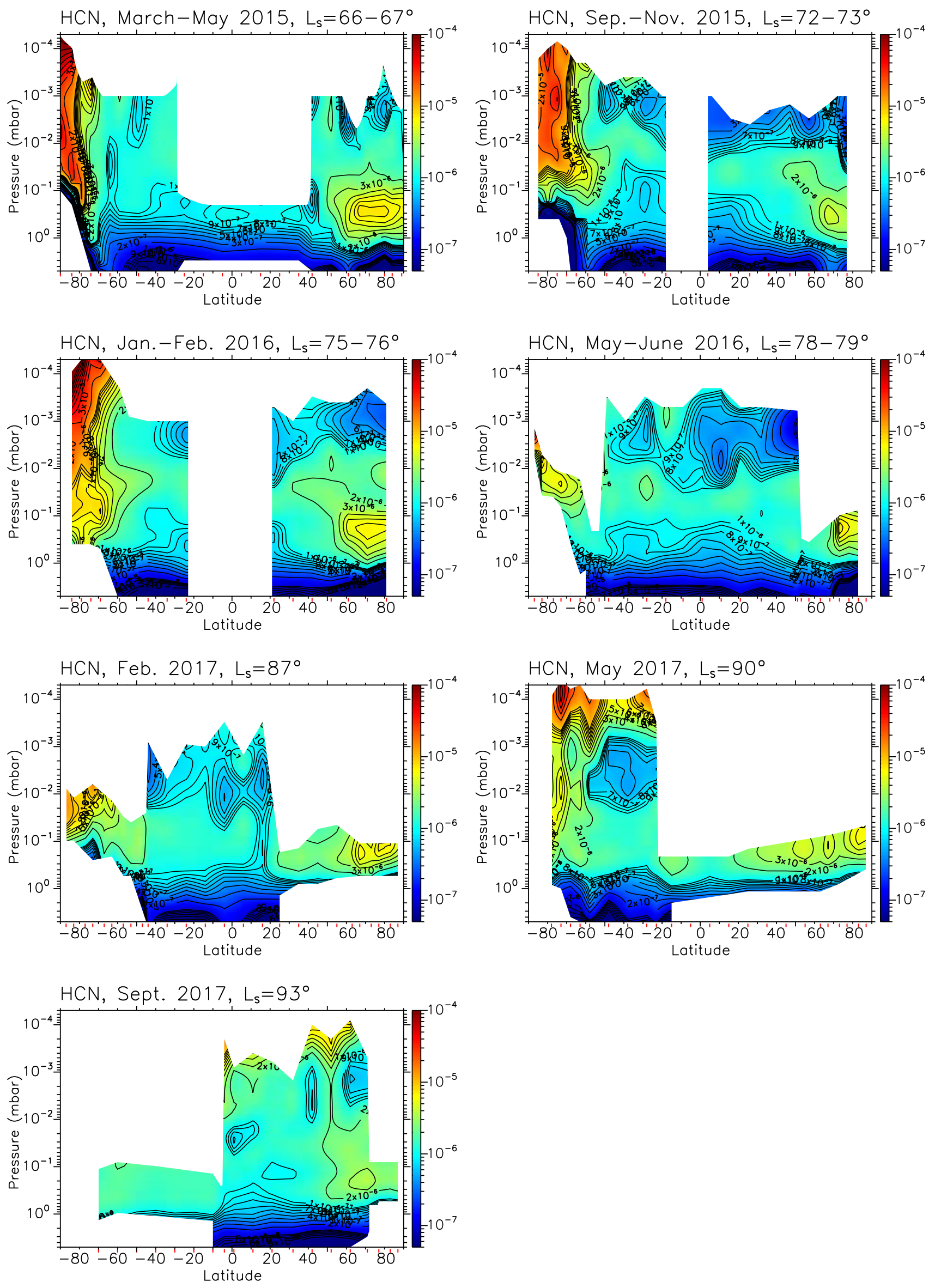

Fig. A.2. Same as Fig. 3, but for HCN. 
S. Vinatier et al.: Seasonal variations in Titan's atmosphere close to the northern summer solstice

$\mathrm{C}_{4} \mathrm{H}_{2}$, March-May 2015, $\mathrm{L}_{s}=66-67^{\circ}$

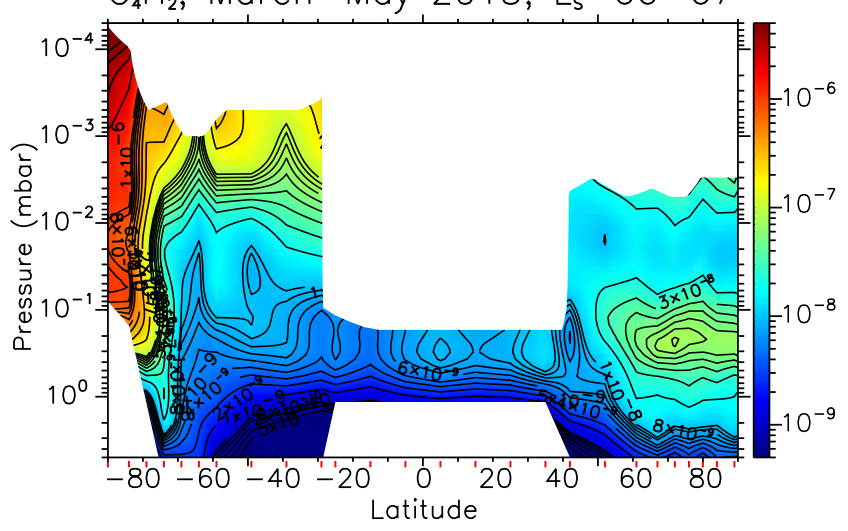

$\mathrm{C}_{4} \mathrm{H}_{2}$, Jan. - Feb. 2016, $\mathrm{L}_{s}=75-76^{\circ}$

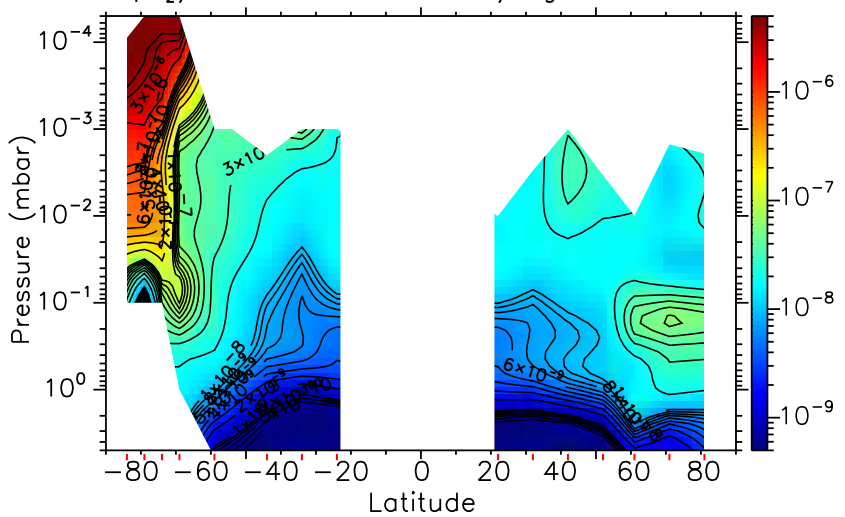

$\mathrm{C}_{4} \mathrm{H}_{2}$, Feb. 2017, $L_{s}=87^{\circ}$

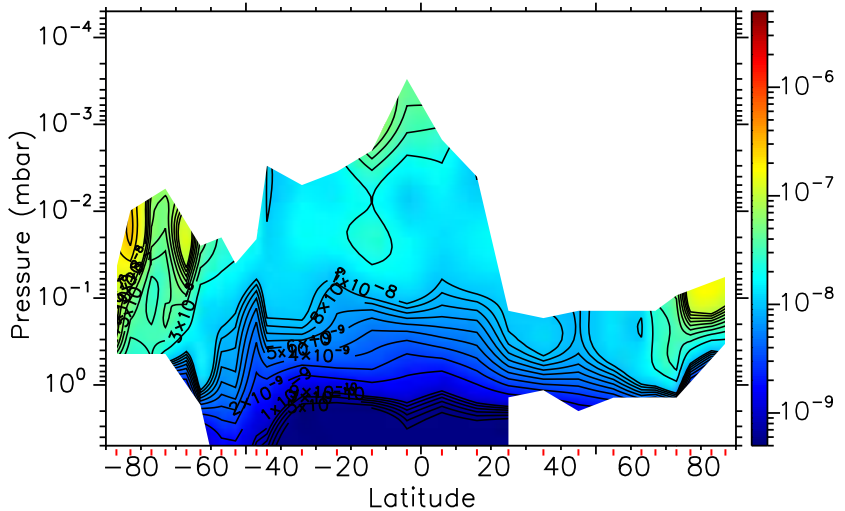

$\mathrm{C}_{4} \mathrm{H}_{2}$, Sept. 2017, $\mathrm{L}_{s}=93^{\circ}$

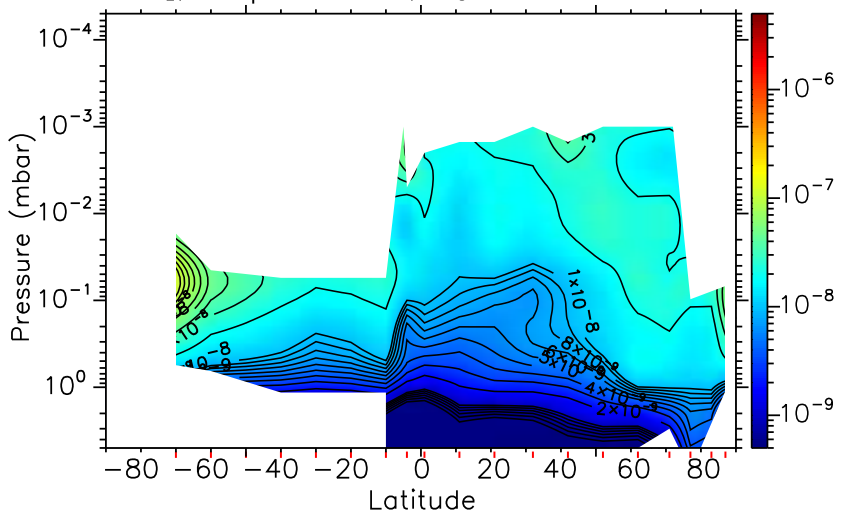

$\mathrm{C}_{4} \mathrm{H}_{2}$, Sep. - Nov. 2015, $\mathrm{L}_{s}=72-73^{\circ}$

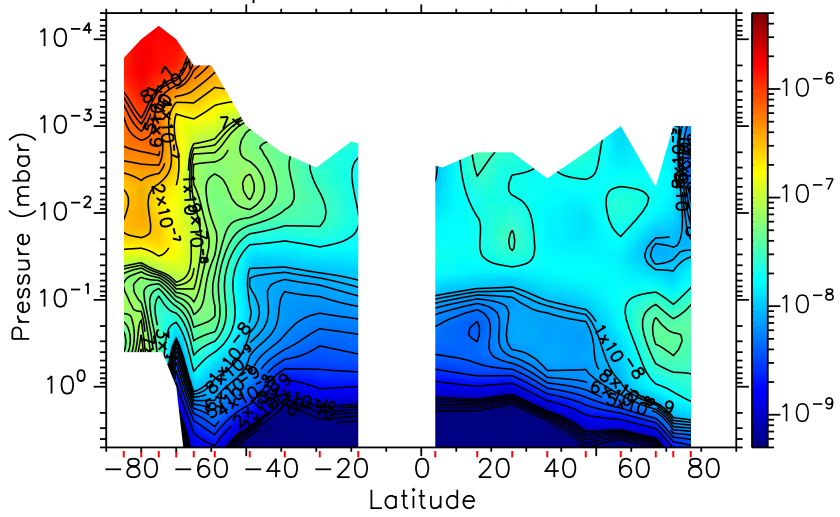

$\mathrm{C}_{4} \mathrm{H}_{2}$, May-June 2016, $\mathrm{L}_{5}=78-79^{\circ}$

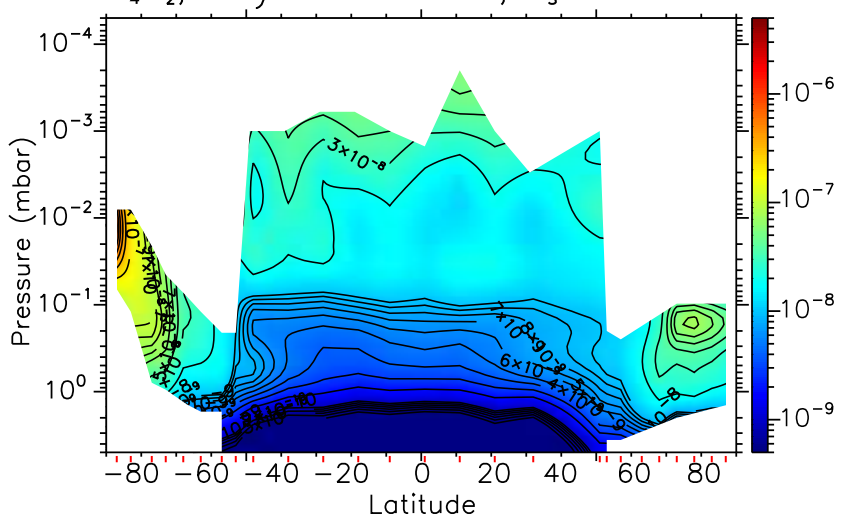

$\mathrm{C}_{4} \mathrm{H}_{2}$, May $2017, L_{s}=90^{\circ}$

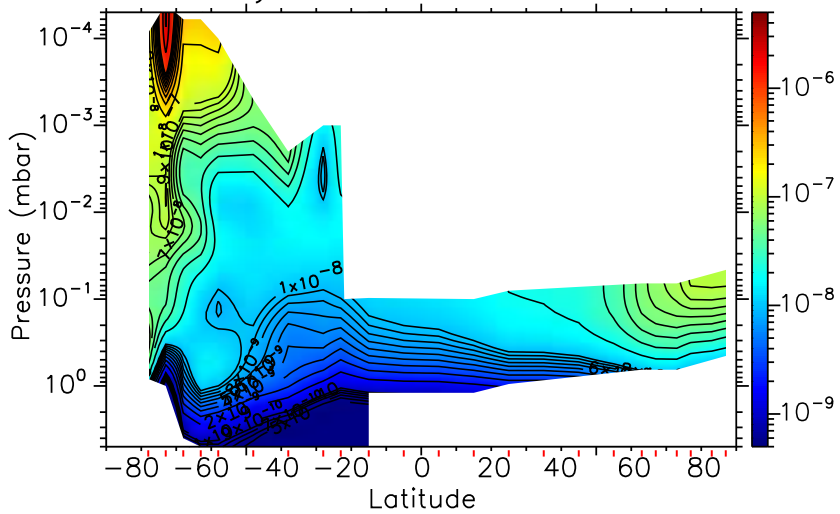

Fig. A.3. Same as Fig. 3, but for $\mathrm{C}_{4} \mathrm{H}_{2}$. 

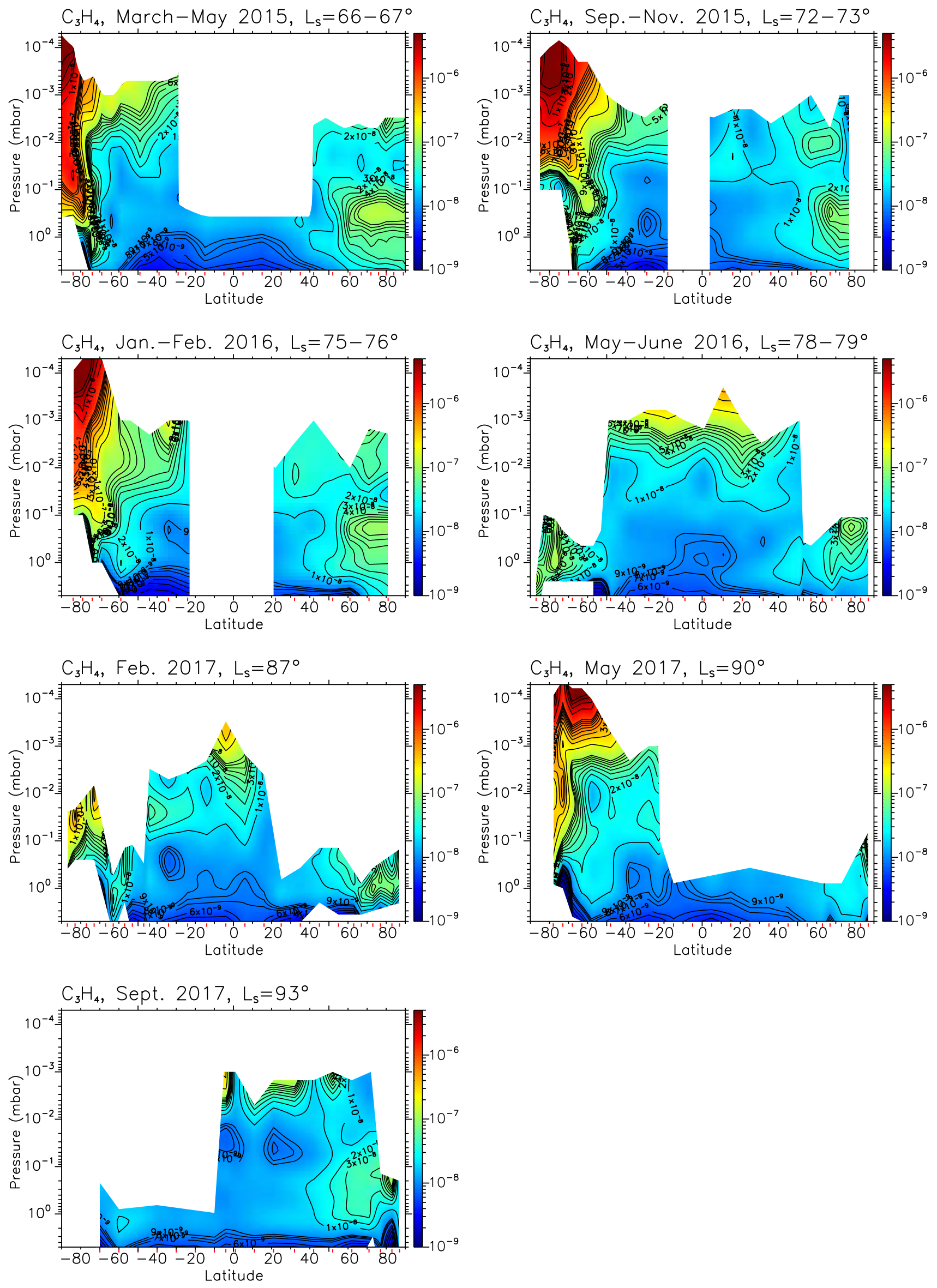

Fig. A.4. Same as Fig. 3, but for $\mathrm{CH}_{3} \mathrm{C}_{2} \mathrm{H}$. 
S. Vinatier et al.: Seasonal variations in Titan's atmosphere close to the northern summer solstice
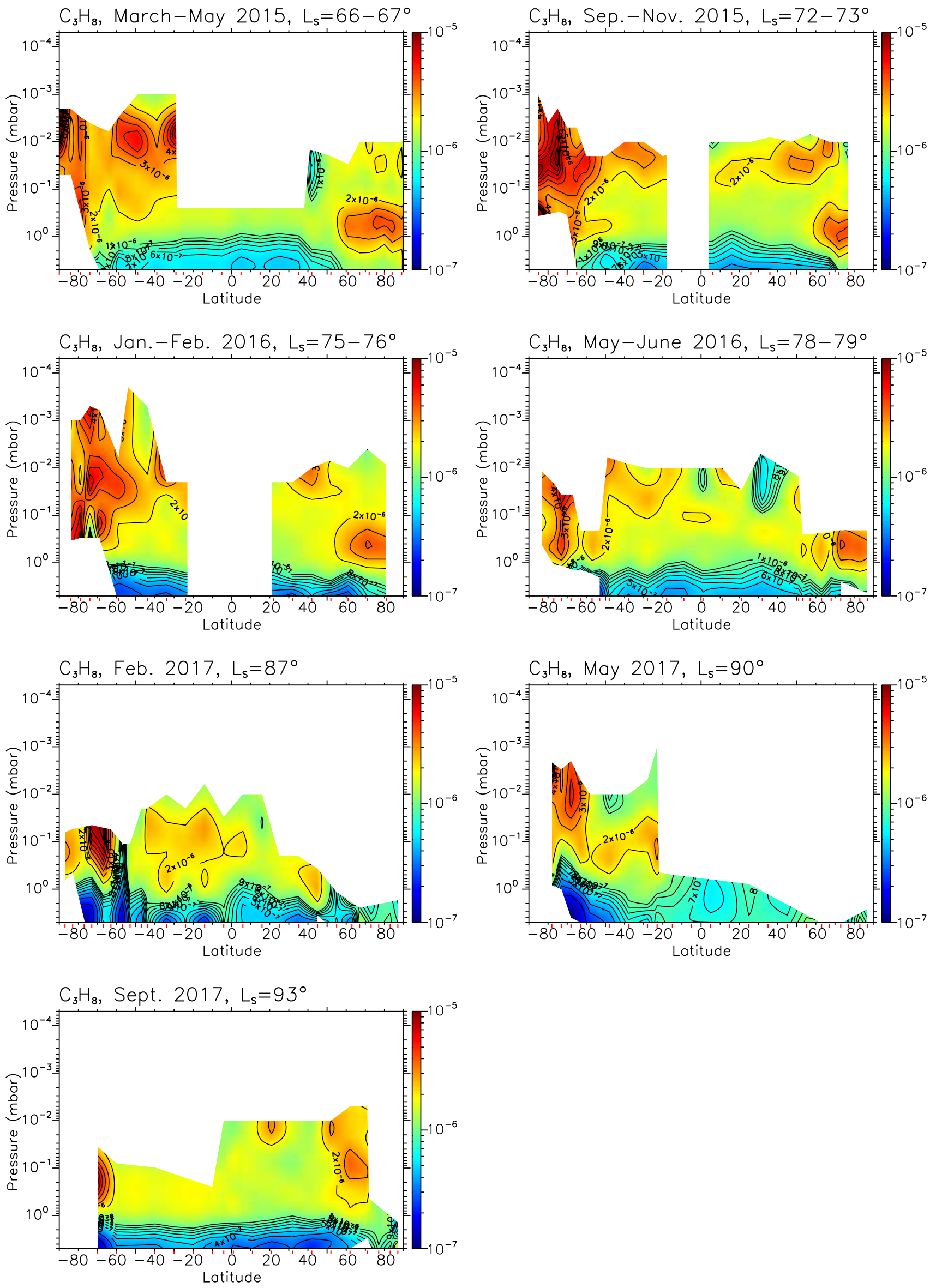

Fig. A.5. Same as Fig. 3, but for $\mathrm{C}_{3} \mathrm{H}_{8}$. 

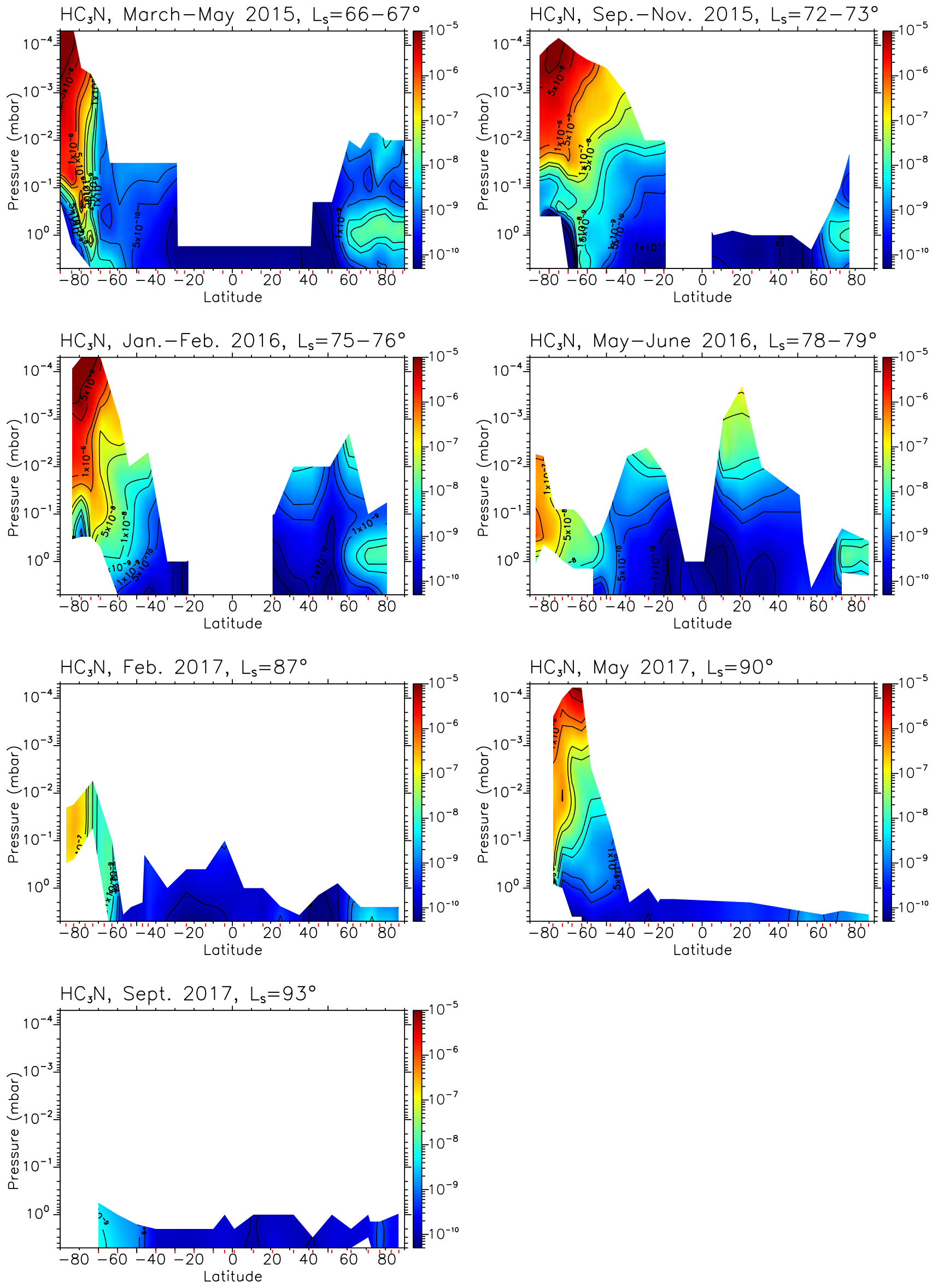

Fig. A.6. Same as Fig. 3, but for $\mathrm{HC}_{3} \mathrm{~N}$. 
S. Vinatier et al.: Seasonal variations in Titan's atmosphere close to the northern summer solstice
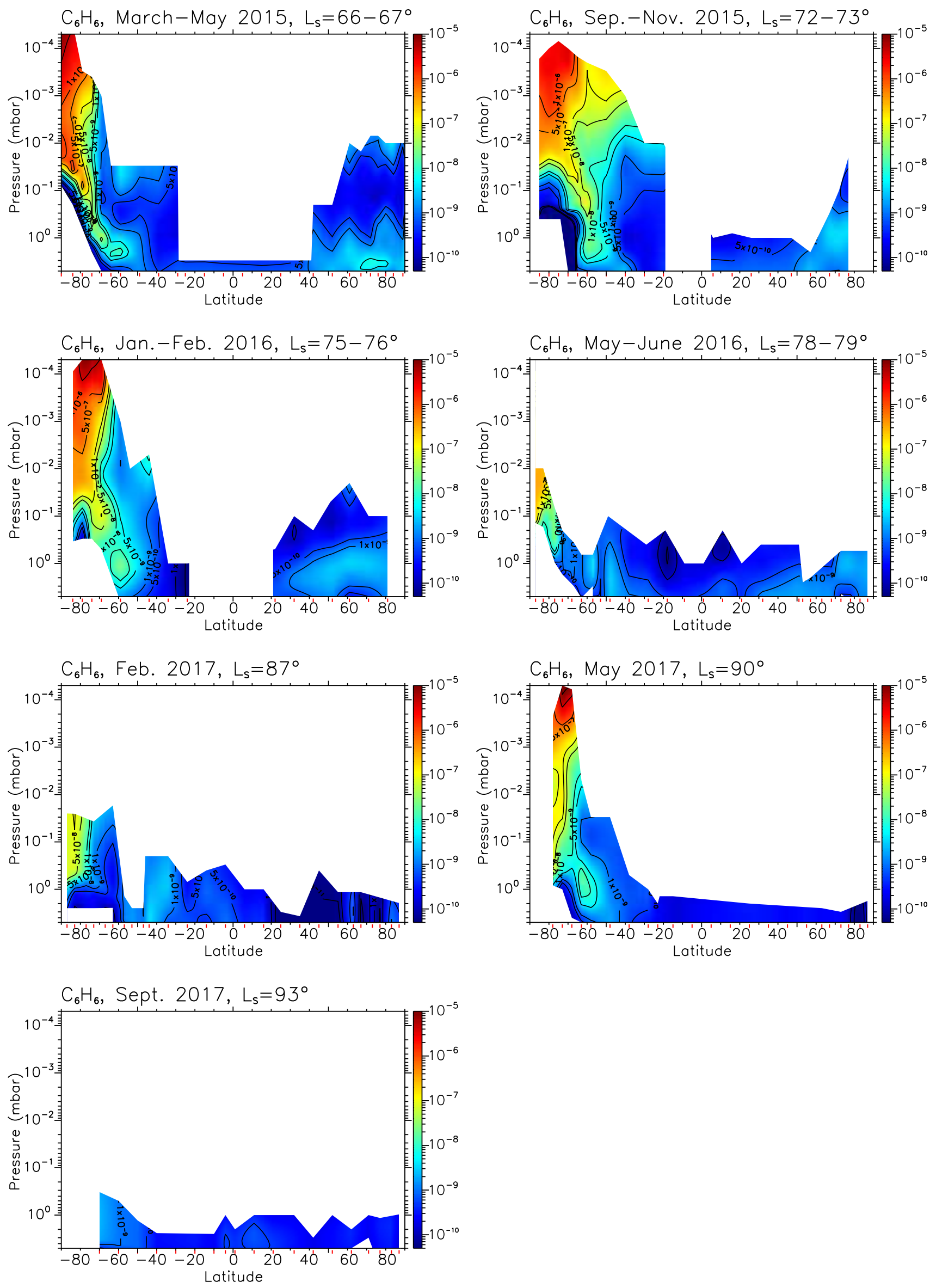

Fig. A.7. Same as Fig. 3, but for $\mathrm{C}_{6} \mathrm{H}_{6}$. 

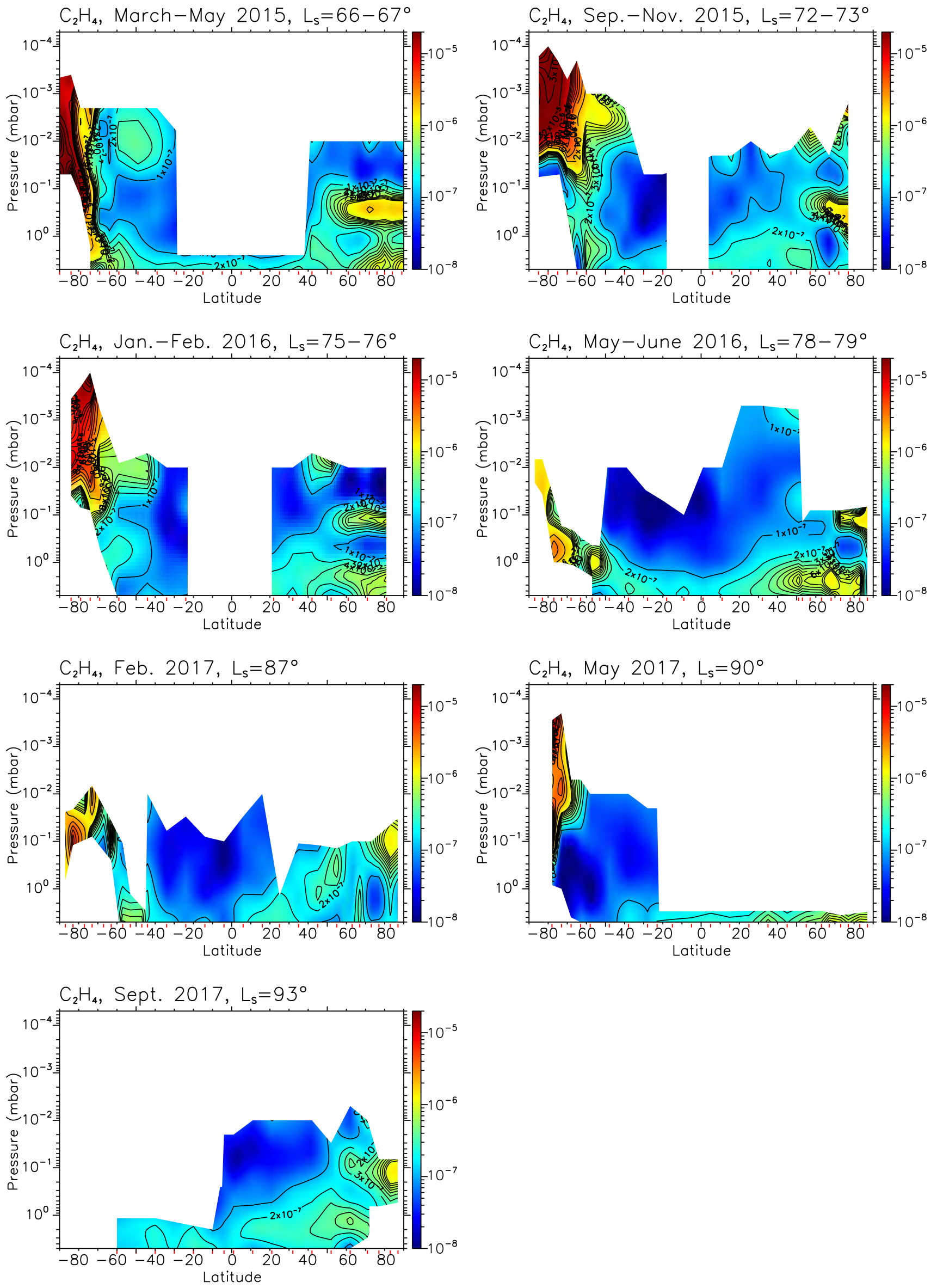

Fig. A.8. Same as Fig. 3, but for $\mathrm{C}_{2} \mathrm{H}_{4}$. 
S. Vinatier et al.: Seasonal variations in Titan's atmosphere close to the northern summer solstice
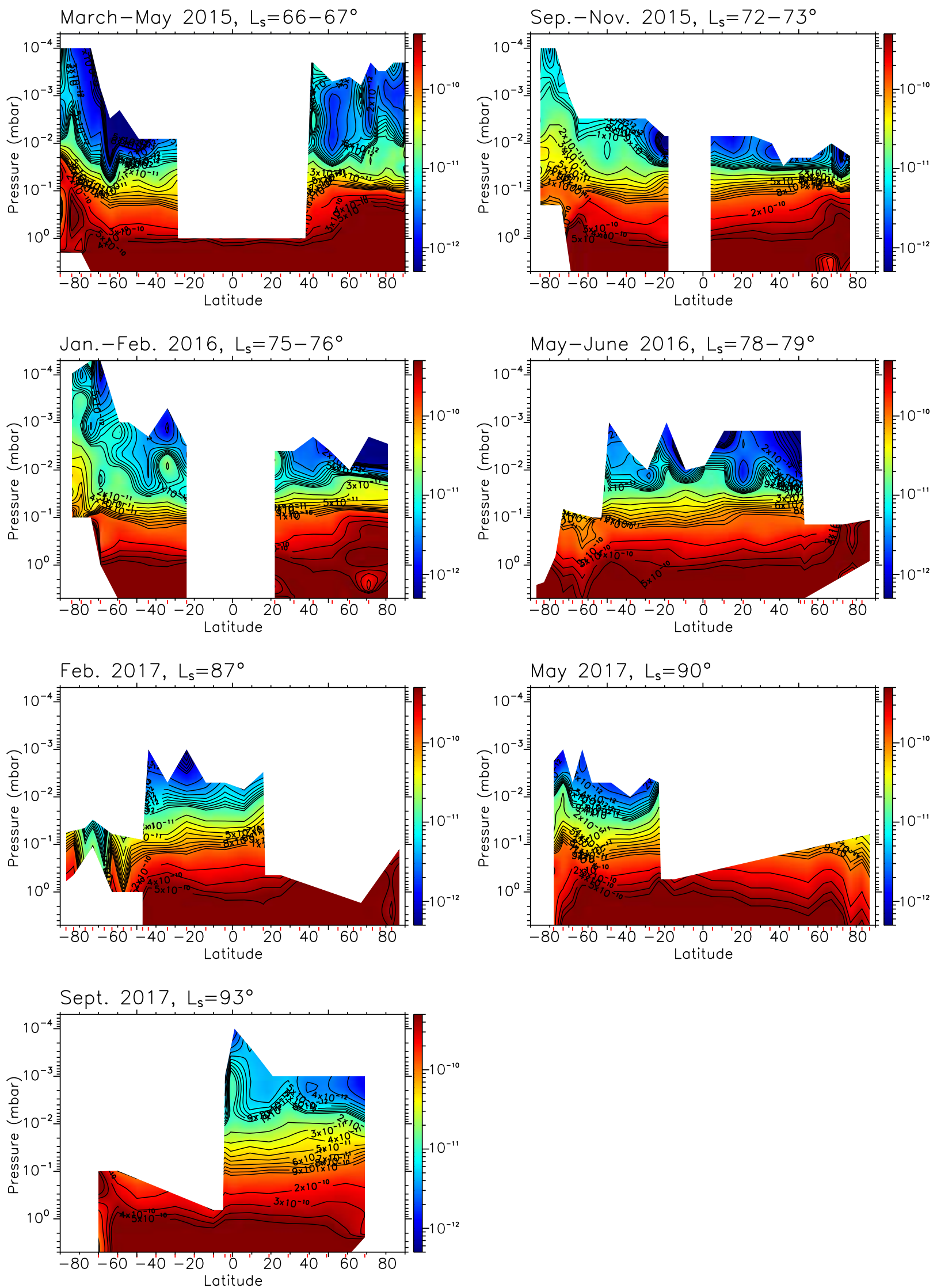

Fig. A.9. Haze extinction coefficient (given in $\mathrm{cm}^{-1}$ and representative of $1090 \mathrm{~cm}^{-1}$ ) meridional cross sections from March 2015 to September 2017. Extinction coefficient is derived from the fit of the $595-660 \mathrm{~cm}^{-1}$ spectral range and scaled to $1090 \mathrm{~cm}^{-1}$ using the spectral dependence derived by Vinatier et al. (2012). Regions without information are represented in white; they correspond to missing data or pressure levels not probed by nadir observations. 


\section{Appendix B: Observations characteristics}

Characteristics of the limb and nadir observations used in this study are listed in Tables B.1 and B.2, respectively.

Table B.1. Characteristics of the averaged FP4 limb spectra.

\begin{tabular}{|c|c|c|c|c|c|c|c|}
\hline Flyby, date & $L_{s}\left({ }^{\circ}\right)$ & $\begin{array}{l}\text { Latitudes } \\
\left({ }^{\circ} \mathrm{N}\right)\end{array}$ & $\begin{array}{l}\text { Longitudes } \\
\left({ }^{\circ} \mathrm{W}\right)\end{array}$ & $\begin{array}{l}\text { Local } \\
\text { time }\end{array}$ & $\operatorname{SZA}\left({ }^{\circ}\right)$ & $\begin{array}{l}\text { Vertical } \\
\text { res. }(\mathrm{km})\end{array}$ & $\begin{array}{l}\text { Vertical shifts }(\mathrm{km}) \text { applied to } \\
\text { the upper/lower pointings }\end{array}$ \\
\hline T110, 2015 Mar. 17 & 65.8 & $\begin{array}{c}-78,-84,-86,-81,-77,-72 \\
-67,-61,-51,-41,-31\end{array}$ & $\begin{array}{c}119,138,210,255,265,270 \\
272,275,278,280,282\end{array}$ & $\begin{array}{c}02: 52,01: 36,20: 55,17: 58,17: 14,16: 55 \\
16: 43,16: 35,16: 25,16: 19,16: 14\end{array}$ & $\begin{array}{c}112,120,117,114,111,108 \\
104,102,95,89,82\end{array}$ & $\begin{array}{c}45,44,43,41,40,39 \\
38,36,34,31,29\end{array}$ & $\begin{array}{l}0 / 0,0 / 15,0 / 24,2 / 3,2 / 3,8 / 38 \\
-6 / 3,-6 / 8,-2 / 16,8 / 14,-8 / 1\end{array}$ \\
\hline T111, 2015 May 9 & 67.4 & $\begin{array}{c}81,86,87,82,78,74 \\
69,64,59,49,39\end{array}$ & $\begin{array}{c}61,50,282,262,259,257 \\
256,256,255,255,255\end{array}$ & $\begin{array}{c}14: 12,15: 00,23: 35,00: 50,01: 10,01: 16 \\
01: 21,01: 24,01: 26,01: 29,01: 31\end{array}$ & $\begin{array}{l}58,63,68,73,77,81 \\
86,90,95,104,113\end{array}$ & $\begin{array}{c}29,31,32,33,34,36 \\
37,39,40,43,46\end{array}$ & $\begin{array}{l}-2 / 36,16 / 30,16 / 14,0 / 7,-2 / 3,-6 / 7 \\
0 / 21,10 / 2,-54 /-118,6 / 30,-22 /-18\end{array}$ \\
\hline T113, 2015 Sep. 29 & 71.7 & $\begin{array}{c}-86,-81,-76,-71,-66 \\
-61,-56,-52,-42,-32,-22\end{array}$ & $\begin{array}{c}80,67,64,62,61 \\
60,60,59,59,58,58\end{array}$ & $\begin{array}{c}12: 37,13: 27,13: 41,13: 47,13: 51 \\
13: 53,13: 55,13: 56,13: 57,13: 58,13: 59\end{array}$ & $\begin{array}{l}111,107,103,98,94 \\
90,85,81,72,63,55\end{array}$ & $\begin{array}{c}29,30,32,34,35 \\
36,37,39,42,44,47\end{array}$ & $\begin{array}{c}0 /-32,0 / 22,0 / 17,0 /-1,0 / 2 \\
0 / 17,18 / 25,2 / 23,2 /-2,-12 / 5,16 / 7\end{array}$ \\
\hline T114, 2015 Nov. 13 & 73.1 & $\begin{array}{c}4,14,24,34,44,54 \\
59,64,69,74\end{array}$ & $\begin{array}{c}306,305,304,303,303,302 \\
302,302,303,304\end{array}$ & $\begin{array}{c}17: 46,17: 48,17: 50,17: 51,17: 52,17: 51 \\
17: 50,17: 49,17: 48,17: 43\end{array}$ & $\begin{array}{c}85,81,78,74,71,68 \\
67,66,65,64\end{array}$ & $\begin{array}{c}28,29,32,35,37,40 \\
42,43,44,46\end{array}$ & $\begin{array}{c}0 / 7,8 / 9,-8 / 20,22 / 19,6 / 28,16 / 18 \\
14 / 30,4 / 26,-8 / 9,-40 / 6\end{array}$ \\
\hline T115, 2016 Jan. 17 & 75.1 & $\begin{array}{l}-86,-81,-76,-71,-66 \\
-61,-56,-\mathrm{y} 46,-36,-27\end{array}$ & $\begin{array}{l}159,141,136,133,132 \\
131,131,130,130,129\end{array}$ & $\begin{array}{l}04: 10,05: 25,05: 47,05: 57,06: 04 \\
06: 08,06: 11,06: 16,06: 19,06: 21\end{array}$ & $\begin{array}{l}118,116,116,114,113 \\
111,110,106,101,97\end{array}$ & $\begin{array}{l}29,31,32,33,35 \\
36,38,40,43,46\end{array}$ & $\begin{array}{c}0 / 0,12 / 24,10 / 31,0 / 2 \\
6 / 18,8 / 21,12 / 23,-42 / 6,-6 / 11\end{array}$ \\
\hline T116, 2016 Feb. 2 & 75.6 & $\begin{array}{c}19,29,39,49 \\
59,69,79\end{array}$ & $\begin{array}{c}308,309,311,313 \\
316,322,339\end{array}$ & $\begin{array}{c}18: 25,18: 18,18: 10,18: 01 \\
17: 47,17: 22,16: 08\end{array}$ & $\begin{array}{c}87,82,76,71 \\
66,63,60\end{array}$ & $\begin{array}{c}46,43,40,37 \\
35,32,29\end{array}$ & $\begin{array}{c}25 / 56,4 / 16,30 / 44,30 / 38 \\
20 / 32,10 / 27,15 / 20\end{array}$ \\
\hline T119, 2016 May 7 & 78.4 & $\begin{array}{c}-51,-41,-31,-21 \\
-11,-1,9,14\end{array}$ & $\begin{array}{c}134,124,117,111 \\
106,102,97,94\end{array}$ & $\begin{array}{l}04: 41,05: 25,05: 56,06: 21 \\
06: 42,07: 02,07: 23,07: 33\end{array}$ & $\begin{array}{c}122,113,104,95 \\
86,77,67,63\end{array}$ & $\begin{array}{l}47,44,42,39 \\
36,33,31,29\end{array}$ & $\begin{array}{c}-10 / 9,-18 / 0,-14 / 13,-6 / 5 \\
8 / 3,-6 / 12,-10 / 14,10 / 13\end{array}$ \\
\hline T120, 2016 Jun. 8 & 79.4 & 29,50 & 327,5 & $16: 42,14: 08$ & 61,34 & 35,29 & $62 / 36,6 / 18$ \\
\hline S98/259TI, 2017 Feb. 2 & 86.6 & $\begin{array}{c}-61,-47,-37,-27 \\
-18,-8,2,12\end{array}$ & $\begin{array}{l}114,66,52,42 \\
24,28,22,18\end{array}$ & $\begin{array}{l}06: 05,09: 22,10: 19,11: 01 \\
11: 35,12: 02,12: 27,12: 48\end{array}$ & $\begin{array}{c}111,82,68,55 \\
45,35,26,19\end{array}$ & $\begin{array}{l}61,61,62,63 \\
64,64,66,68\end{array}$ & $\begin{array}{l}28 /-8,18 /-8,-2 /-31,24 / 38 \\
8 / 17,68 / 66,-4 /-6,-34 /-13\end{array}$ \\
\hline S99/275TI, 2017 May 25 & 90.0 & $\begin{array}{c}-82,-77,-72,-67,-62 \\
-52,-42,-32,-28\end{array}$ & $\begin{array}{l}30,20,15,12,11 \\
8,6,5,5\end{array}$ & $\begin{array}{c}11: 13,11: 54,12: 12,12: 25,12: 34 \\
12: 46,12: 54,13: 00,13: 03\end{array}$ & $\begin{array}{c}109,104,99,94,89 \\
79,70,61,56\end{array}$ & $\begin{array}{c}51,52,54,55,57 \\
59,62,65,66\end{array}$ & $\begin{array}{c}32 / 5,20 / 47,36 / 72,40 / 60,6 / 32 \\
4 / 22,6 / 6,22 / 64,6 / 5\end{array}$ \\
\hline S101/292TI, 2017 Sep. 11 & 93.3 & $\begin{array}{l}-6,-1,9,19,29 \\
39,49,59,69\end{array}$ & $\begin{array}{c}183,186,190,193,197 \\
200,204,212,230\end{array}$ & $\begin{array}{c}22: 47,22: 35,22: 16,22: 00,21: 45 \\
21: 30,21: 10,20: 39,19: 27\end{array}$ & $\begin{array}{c}153,147,137,126,116 \\
106,96,85,72\end{array}$ & $\begin{array}{c}39,40,42,43,45 \\
48,50,52,54\end{array}$ & $\begin{array}{c}42 / 34,4 / 45,24 / 29,30 / 24,0 / 23 \\
14 / 26,-8 / 34,30 / 6,10 / 49\end{array}$ \\
\hline
\end{tabular}

Notes. The first and second columns give the Titan flyby number with its date and the corresponding planetocentric orbital longitude of the Sun, respectively. The third and fourth columns give the latitude and longitude of each limb observation used to infer global maps of temperature, aerosol extinction, and molecular mixing ratio. The fifth column gives the mean local time of each observation, the sixth column gives the solar zenith angle and the seventh column gives the vertical resolution. The last column gives the vertical shifts applied to the line-of-sight altitudes of the deepest and the upper pointing positions of each limb observation. Spectra were extracted from the "global calibration" version of the CIRS database (Jennings et al. 2017).

Table B.2. Characteristics of the averaged FP4 nadir spectra.

\begin{tabular}{ccccc}
\hline \hline Flyby, date & $L_{s}\left({ }^{\circ}\right)$ & Latitudes $\left({ }^{\circ} \mathrm{N}\right)$ & Longitudes $\left({ }^{\circ} \mathrm{W}\right)$ & Emission angle \\
\hline T107, 2014 Dec. 10 & 62.8 & $-35,-25,-17$ & $165,176,164$ & $15,12,17$ \\
T108, 2015 Jan. 12 & 63.8 & $-4,9,25,35$ & $213,14,12,210$ & $24,32,48,25$ \\
T119, 2016 May 5 & 78.4 & $-85,-79,-74,-69,-63,-58,-48$ & $157,179,178,186,163,165,140$ & $62,56,51,46,41,36,26$ \\
T120, 2016 June 8 & 79.4 & $48,54,58,63,69,73,79,84$ & $220,220,218,220,217,210,208,207$ & $11,17,22,26,32,37,42,46$ \\
S98/259TI, 2017 Feb. 1 & 86.6 & $-44,-49,-52,-59,-y 64$ & $283,288,241,112,67$ & $12,12,15,30,35$ \\
& & $-68,-74,-82,-85$ & $78,47,139,200$ & $34,45,51,48$ \\
S98/262TI, 2017 Feb. 17 & 87.1 & $24,33,43,53,61$ & $100,104,110,114,110$ & $8,15,25,36,45$ \\
& & $66,71,76,81,85$ & $110,111,111,123,164$ & $50,54,59,65,69$ \\
S100/278TI, 2017 June 8-9 90.4 & $-9,0,11,21,31,39,48$ & $75,49,27,32,35,50,41$ & $67,56,43,33,23,12,6$ \\
& & $56,65,69,74,79,83,86$ & $36,68,48,78,104,182,153$ & $7,15,18,26,31,39,41$ \\
S101/293TI, 2017 Sept. 12 & 93.3 & $-64,-58,-44,-39,-29,-21,-11$ & $96,72,72,72,75,68,67$ & $79,73,57,52,42,37,25$ \\
& & $70,73,81,85$ & $134,169,108,146$ & $22,12,22,24$ \\
\hline
\end{tabular}

Notes. The first column gives the Titan flyby number and its date, the second gives the planetocentric orbital longitude of the Sun at the date of the flyby. The third and fourth columns respectively give the mean latitudes and longitudes of each averaged nadir selected spectra. The last column gives the mean emission angle. Spectra were extracted from the 4.3.5 version of the CIRS database. 


\section{Appendix C: Altitude-latitude cross sections of the thermal field and photochemical species distribution}

Figures C.1-C.4 give another way to display the thermal field (Fig. C.1) and the mixing ratios of trace gas distributions (Figs. C.1-C.4) with the altitude as vertical axis and on a polar projection with the north pole at the top and south pole at the bottom of each panel. This more compact visualization allows us to plot the distributions derived for all the studied dates in a single row. It is then possible to compare the spatial distributions of up to three molecules in a single figure. Color scales are the same as those in Figs. 2 to A.8. Additionally, Fig. C.4 displays a comparison of the haze mass mixing ratio distributions derived from the spectral continuum around $610 \mathrm{~cm}^{-1}$ (FP3, middle) and $1090 \mathrm{~cm}^{-1}$ (FP4, bottom). The main structures derived from the two spectral ranges are very similar except at altitudes higher than $400 \mathrm{~km}$, where uncertainties on the continuum radiance become important. 

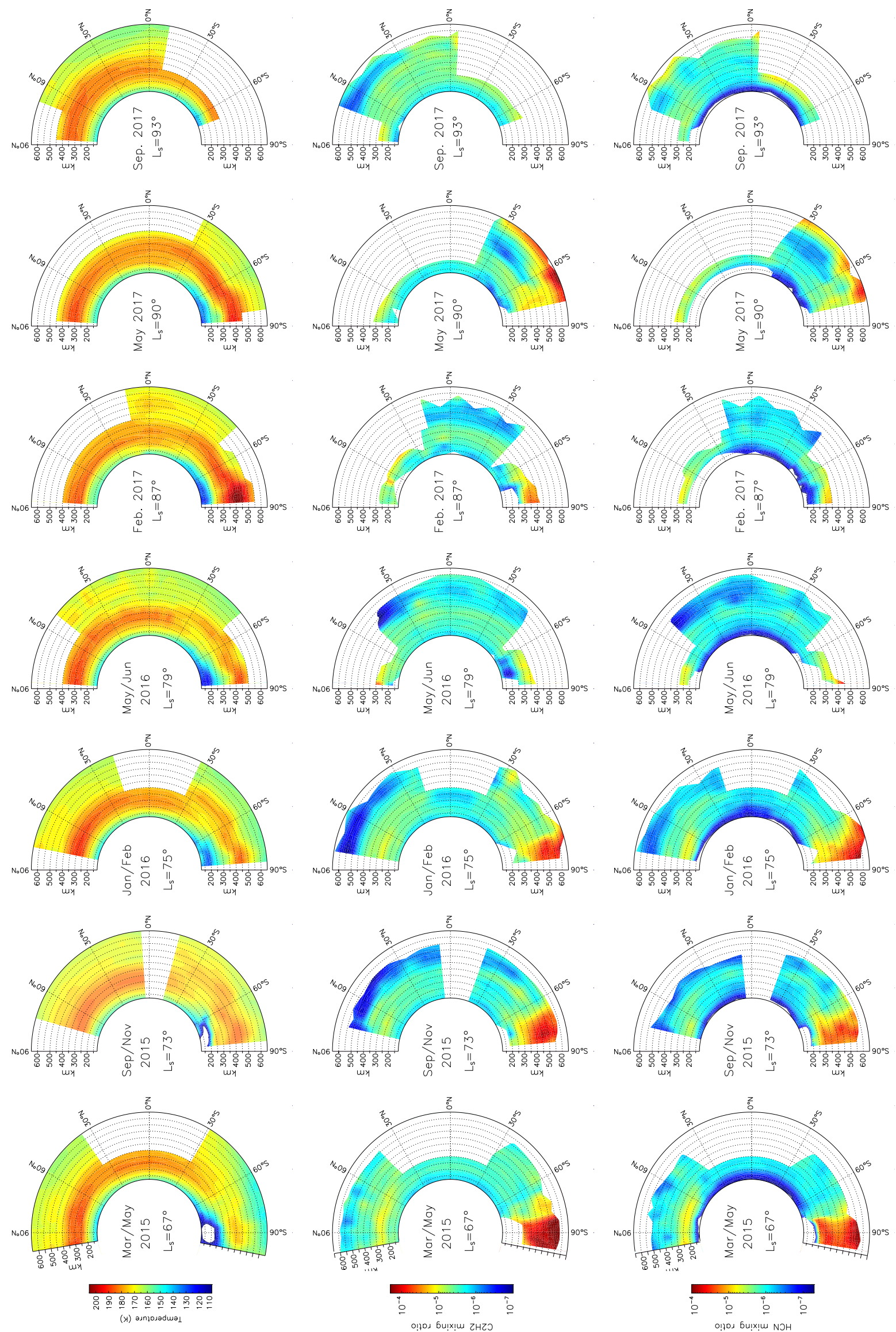

Fig. C.1. Thermal field (top), $\mathrm{C}_{2} \mathrm{H}_{2}$ (middle), and $\mathrm{HCN}$ (bottom) volume mixing ratio meridional cross sections from March 2015 to September 2017 (from left to right). 
S. Vinatier et al.: Seasonal variations in Titan's atmosphere close to the northern summer solstice
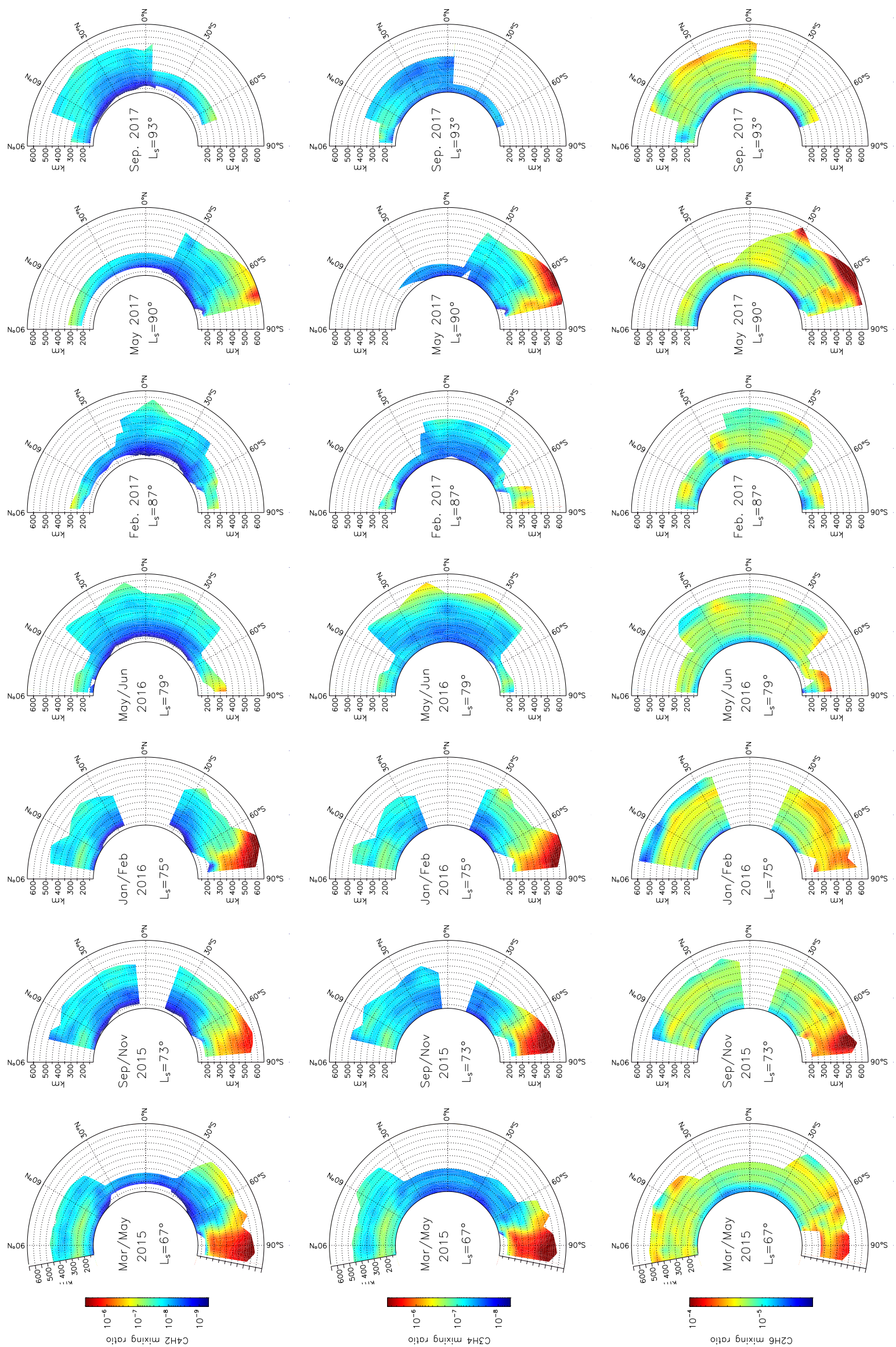

Fig. C.2. $\mathrm{C}_{4} \mathrm{H}_{2}$ (top), $\mathrm{CH}_{3} \mathrm{C}_{2} \mathrm{H}$ (middle), and $\mathrm{C}_{2} \mathrm{H}_{6}$ (bottom) volume mixing ratio meridional cross sections seasonal changes from March 2015 to September 2017 (from left to right). 

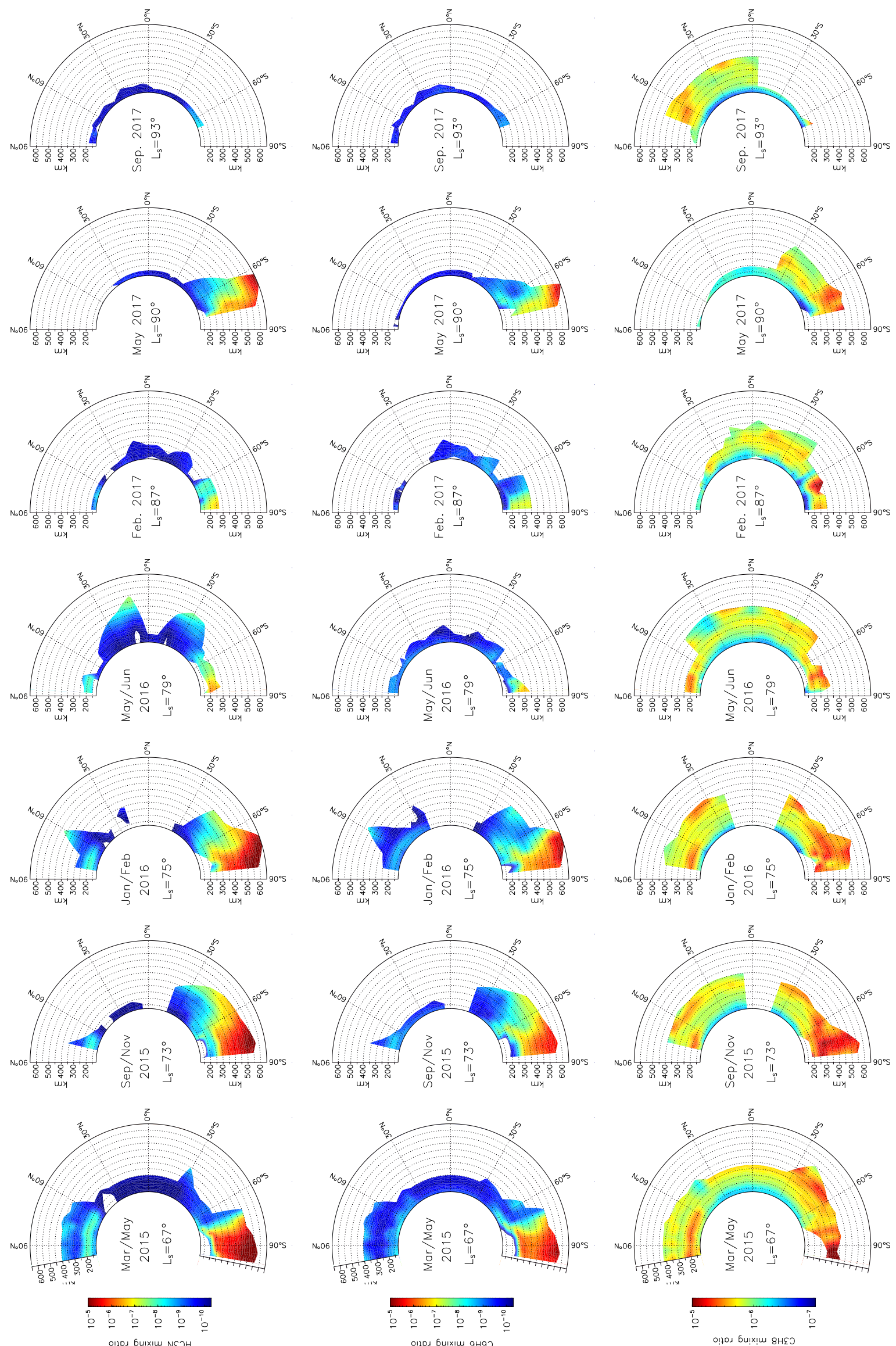

Fig. C.3. $\mathrm{HC}_{3} \mathrm{~N}$ (top), $\mathrm{C}_{6} \mathrm{H}_{6}$ (middle), and $\mathrm{C}_{3} \mathrm{H}_{8}$ (bottom) volume mixing ratio meridional cross sections seasonal changes from March 2015 to September 2017 (from left to right). 
S. Vinatier et al.: Seasonal variations in Titan's atmosphere close to the northern summer solstice
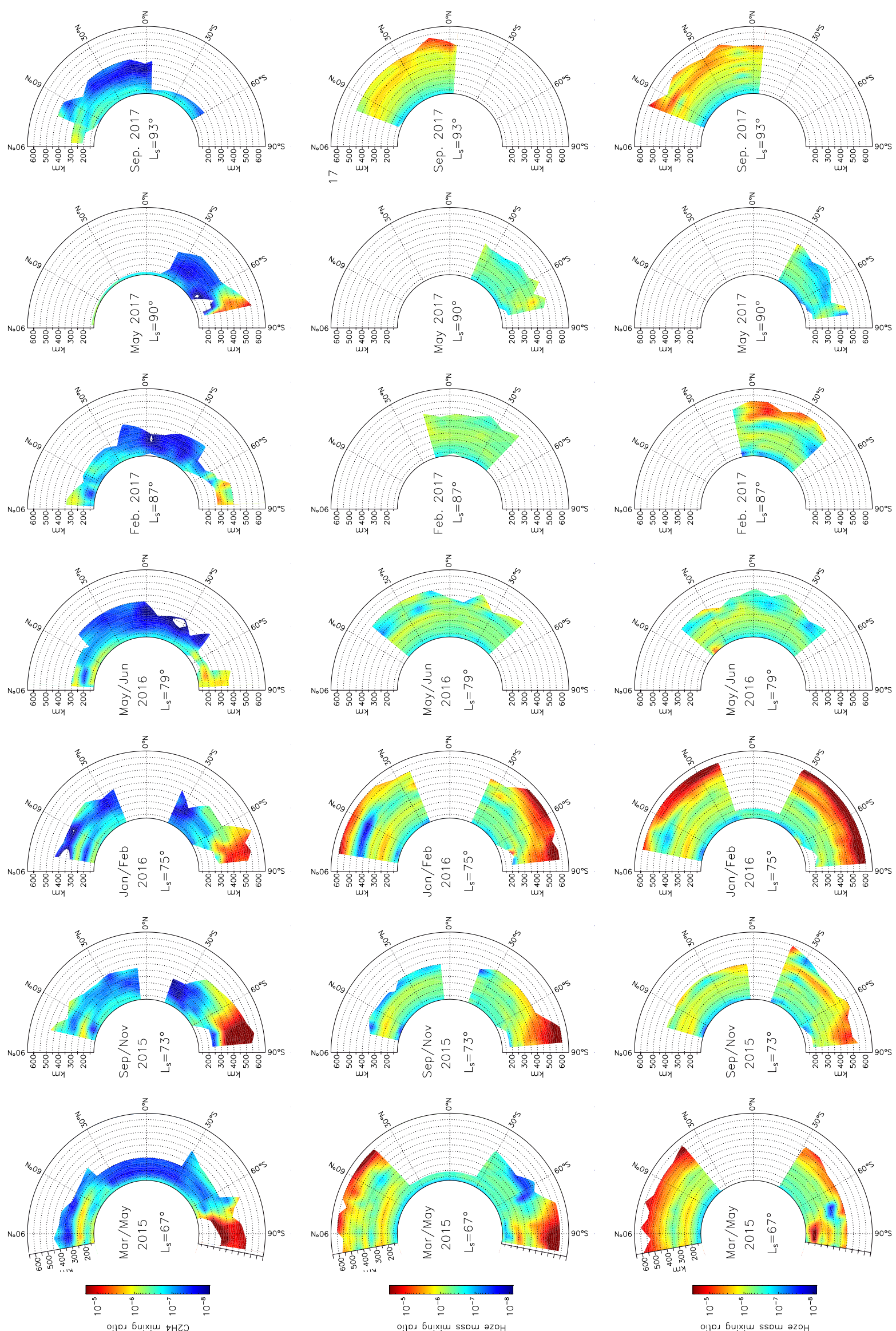

Fig. C.4. Meridional cross sections of $\mathrm{C}_{2} \mathrm{H}_{4}$ volume mixing ratio (top) and haze mass mixing ratio determined from the continuum in the spectral region around $610 \mathrm{~cm}^{-1}\left(\mathrm{FP} 3\right.$, middle) and $1090 \mathrm{~cm}^{-1}$ (FP4, bottom) from March 2015 to September 2017 (from left to right). 


\section{Appendix D: Comparison of our derived meridional cross section of haze extinction with those derived from Cassini/ISS data}

Figures D.1 and D.2 show the meridional cross sections of haze extinction derived in this study (left) compared to extinction inferred from Cassini ISS Narrow Angle camera observations at $338 \mathrm{~nm}$ (Seignovert et al. 2020).
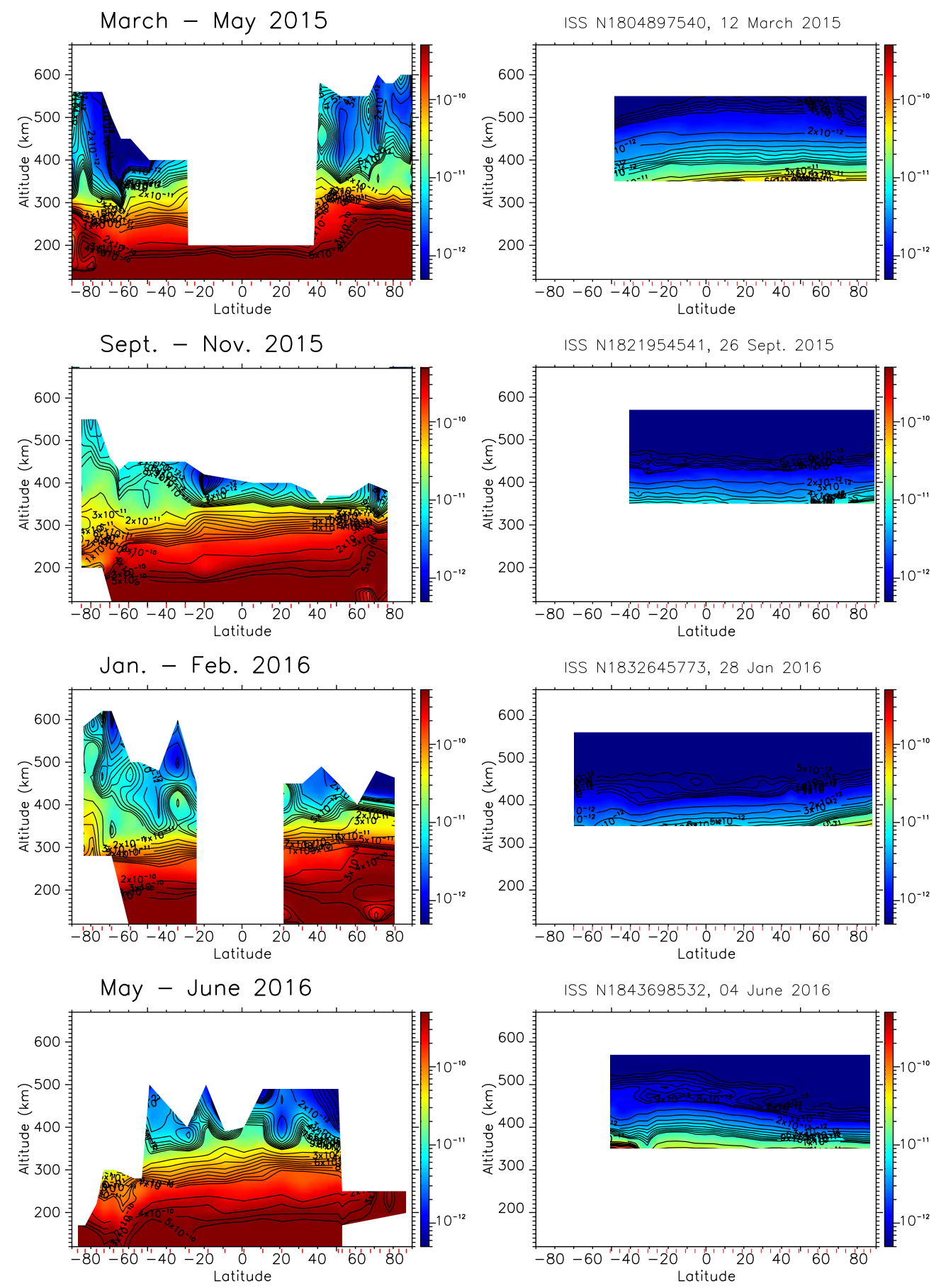

Fig. D.1. Comparison of the aerosol extinction coefficient at $1090 \mathrm{~cm}^{-1}$ derived from CIRS observations in 2015 and 2016 (left column) with those inferred from Cassini ISS Narrow Angle camera at $338 \mathrm{~nm}$ (right column) (Seignovert et al. 2020). Extinction is given in $\mathrm{cm}^{-1}$ and a factor $1.22 \times 10^{-3}$ was applied to the extinction coefficient of Seignovert et al. (2020) in order to take into account the aerosol extinction spectral dependence between $338 \mathrm{~nm}$ and $1090 \mathrm{~cm}^{-1}$ (Vinatier et al. 2012). 
Feb. 2017

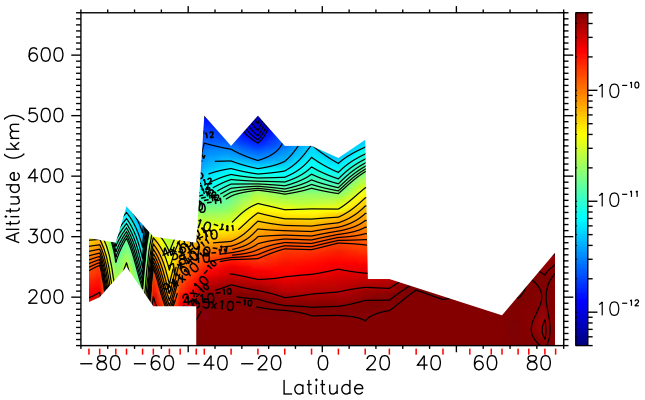

May 2017

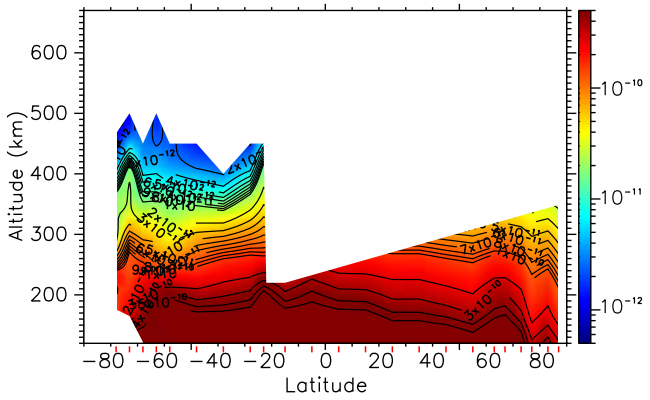

Sept. 2017

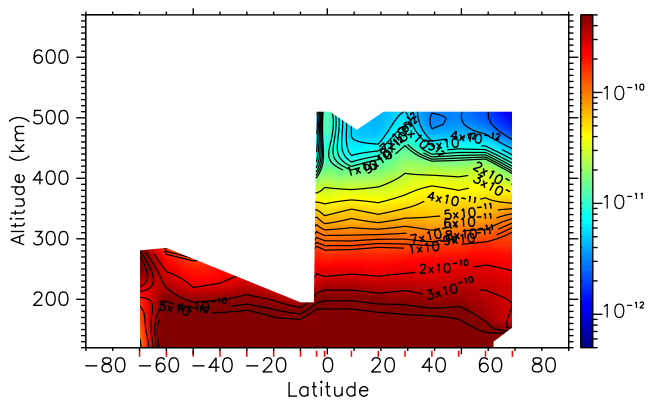

Fig. D.2. Same as Fig. D.1, but for 2017.

\section{Appendix E: Integration of the thermal windshear equation}

Integration of the thermal wind equation is performed over atmospheric parcels of altitude thickness $\delta z$, small compared to the pressure scale height (typically $40 \mathrm{~km}$ in Titan's middle atmosphere) and over the latitude range $\delta \phi$. In the case of Titan a scale study of the projected momentum equations over a northward horizontal $y$-axis, with $\mathrm{d} y=\left(R_{T}+z\right) d \phi=r \mathrm{~d} \phi$, where $R_{T}$ is Titan's radius, $z$ and $\phi$ are the considered altitude and latitude, respectively, leading to the equation

$2 \Omega \sin (\phi) u+\frac{\tan (\phi)}{r} u^{2}+\left.\frac{1}{\rho r} \frac{\partial p}{\partial \phi}\right|_{z}=0$

where $u$ is the zonal wind velocity (eastward), $\Omega$ the rotation rate of Titan $\left(4.56 \times 10^{-6} \mathrm{rad} \mathrm{s}^{-1}\right), z$ the altitude, $\phi$ the latitude, $p$ the pressure, and $\rho$ the atmospheric density. The frictional force component is neglected as it mostly has an impact inside the boundary layer. Deriving Eq. (E.1) with respect to $z$ combined with the ideal gas law leads to

$2 \Omega \sin (\phi) \frac{\partial(u r)}{\partial z}+\tan (\phi) \frac{\partial u^{2}}{\partial z}+\frac{R}{M} \frac{\partial}{\partial z}\left(\left.T \frac{\partial \ln (p)}{\partial \phi}\right|_{z}\right)=0$,

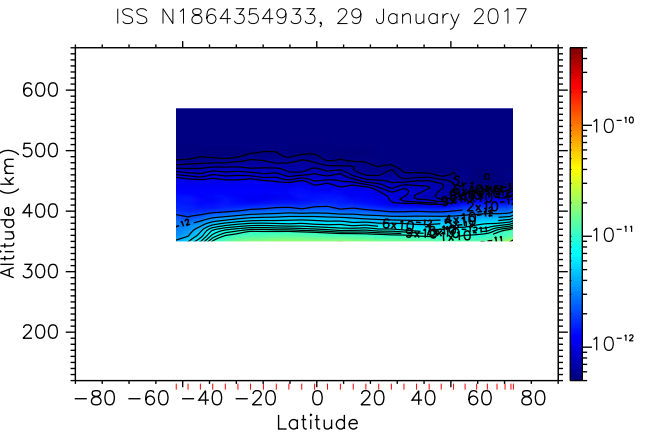

ISS N1873063725, 9 May 2017

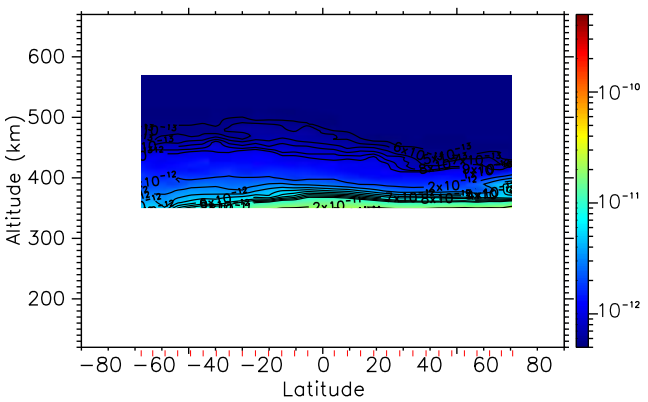

ISS N1884018021, 13 Sep 2017

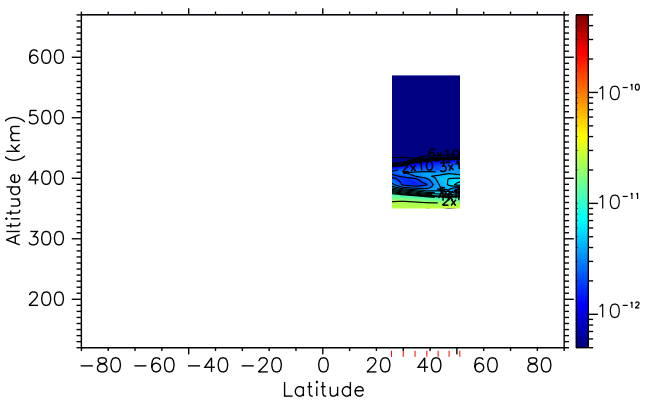

where $M$ is the mean molecular mass, $T$ the temperature, and $R$ the gas constant.

Integration of Eq. (E.2) over the $j$ th layer of altitude range $\left[z_{j}, z_{j+1}\right]$ yields

$$
\begin{aligned}
& 2 \Omega \sin (\phi)\left(r_{j+1} u_{j+1}(\phi)-r_{j} u_{j}(\phi)\right)+\tan (\phi)\left(u_{j+1}^{2}(\phi)-u_{j}^{2}(\phi)\right) \\
& +\frac{R}{M}\left(T_{j+1}(\phi) \frac{\partial \ln \left(p_{j+1}\right)}{\partial \phi}-T_{j}(\phi) \frac{\partial \ln \left(p_{j}\right)}{\partial \phi}\right)=0
\end{aligned}
$$

with the solutions:

$u\left(z_{j+1}, \phi\right)=-\Omega \cos (\phi) r_{j+1} \pm \sqrt{\Omega^{2} \cos ^{2}(\phi) r_{j+1}^{2}-C}$

with $\quad C=\frac{1}{\tan (\phi)} \frac{R}{M}\left[T_{j+1}(\phi) \frac{\partial \ln \left(p_{j+1}\right)}{\partial \phi}-T_{j}(\phi) \frac{\partial \ln \left(p_{j}\right)}{\partial \phi}\right]-u_{j}^{2}-$ $2 \Omega \cos (\phi) r_{j} u_{j}$. wind.

The plus sign (+) in the solution must be applied for eastward

Because of the term $\frac{1}{\tan (\phi)}$, which amplifies any errors on the pressure field (or temperature field) at low latitude, we only considered latitudes higher than $10^{\circ}$. 